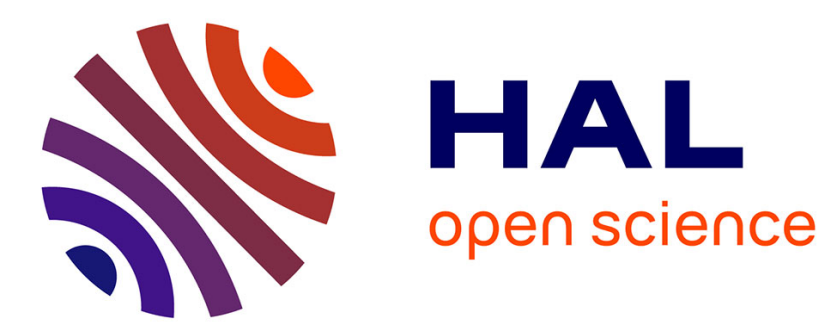

\title{
Regular economies with ambiguity aversion
}

Noé Biheng, Jean-Marc Bonnisseau

\section{To cite this version:}

Noé Biheng, Jean-Marc Bonnisseau. Regular economies with ambiguity aversion. Journal of Mathematical Economics, 2015, 59, pp.24-36. 10.1016/j.jmateco.2015.04.004 . halshs-01185486

\section{HAL Id: halshs-01185486 \\ https://shs.hal.science/halshs-01185486}

Submitted on 20 Aug 2015

HAL is a multi-disciplinary open access archive for the deposit and dissemination of scientific research documents, whether they are published or not. The documents may come from teaching and research institutions in France or abroad, or from public or private research centers.
L'archive ouverte pluridisciplinaire HAL, est destinée au dépôt et à la diffusion de documents scientifiques de niveau recherche, publiés ou non, émanant des établissements d'enseignement et de recherche français ou étrangers, des laboratoires publics ou privés. 


\title{
Regular economies with ambiguity aversion*
}

\author{
Noé Biheng ${ }^{\dagger}$
}

\author{
Jean-Marc Bonnisseau
}

May 15, 2015

\begin{abstract}
We consider a family of exchange economies with complete markets where consumers have multiprior preferences representing their ambiguity aversion. Under a linear independence assumption, we prove that regular economies are generic. Regular economies exhibit enjoyable properties: odd finite number of equilibrium prices, local constancy of this number, local differentiable selections of the equilibrium prices.

Thus, even if ambiguity aversion is represented by non-differentiable multiprior preferences, economies retain generically the properties of the differentiable approach.
\end{abstract}

Keywords: demand function, general equilibrium, ambiguity aversion, multiprior preferences, regular economies, Lipschitz behavior.

JEL Classification: C6, D4, D5.

\section{Introduction}

$\mathbb{P}$ Classically, the global analysis of the general economic equilibrium is based upon well known differential techniques. Basically, one requires the differentiability of the demand functions. We refer the reader to Debreu [11], Mas Colell [22] and Balasko [1] for more details.

*This work was supported by the French National Research Agency, through the program Investissements d'Avenir, ANR-10-LABX-93-01. We wish to thank A. Chateauneuf for his corrections and his valuable comments and an anonymous referee for his remarks and the questions that he raised. All remaining errors are ours.

${ }^{\dagger}$ Paris School of Economics, Université Paris 1 Panthéon Sorbonne, 106-112 boulevard de l'Hôpital 75647 Paris Cedex 13, Noe.Biheng@univ-paris 1.fr

${ }^{\ddagger}$ Paris School of Economics, Université Paris 1 Panthéon Sorbonne, 106-112 boulevard de l'Hôpital 75647 Paris Cedex 13, Jean-marc.Bonnisseau@univ-paris1.fr 
This differentiability is often derived from well known assumptions on the utility functions. Indeed, the utility functions are supposed to be $C^{2}$ to obtain $C^{1}$ demand functions. This does not allow the presence of kinks on indifference curves that arise in uncertainty context.

In the maxmin expected utility model due to Gilboa and Schmeidler [17], the agents face ambiguity modeled by the multiplicity of the priors of the agents. Each agent considers the minimum expected utility over his set of priors. This "minimum" generates kinks on the indifference curves when more than one probability realize the minimum, this leads to the nondifferentiability of the demand functions. These kinks cannot be removed since they are genuinely linked to uncertainty not to modelling issues. The main objective of this paper is to get the genericity of regular economies despite that the demand functions are non-differentiable.

In this paper, we consider an exchange economy with a finite number $\ell$ of commodities and a finite number $m$ of consumers. The preferences of consumer $i$ are represented by a utility function $u_{i}$ from $\mathbb{R}_{++}^{\ell}$ to $\mathbb{R}$. The function $u_{i}$ is the minimum of a finite number $n_{i}$ of expected utility functions that satisfy the usual differentiability requirements and a linear independence assumption on the extremal priors. For example, this linear independence assumption is satisfied by $\varepsilon$-contamination of confidence.

We first study the properties of the demand functions. This systematic study constitutes in itself a new result concerning consumers with multiprior preferences. Indeed, we prove that the demand functions are locally Lipschitz continuous and that these functions are continuously differentiable on an open set of full Lebesgue measure.

In the second part of the paper, we follow Balasko's program. We define and parametrize the equilibrium manifold. We show that it is indeed a smooth manifold at almost every point. As in the classical case, we can propose a global parametrization from which we deduce that the equilibrium manifold is lipeomorphic ${ }^{1}$ to an open connected subset of an Euclidean space denoted by $\mathcal{U}$ using similar approach than Bonnisseau and Rivera-Cayupi [5].

We can define an extended projection using the parametrization. This mapping is continuously differentiable almost everywhere and locally Lipschitz continuous.

Contrary to the classical case, we have to take into account the kinks to define regular economies. A singular economy is either the image of a point where the extended projection is not differentiable or the image of a point

\footnotetext{
${ }^{1}$ Two sets are lipeomorphic if it exists a one-to-one, onto and locally Lipschitz continuous mapping from the first set to the second one with a locally Lipschitz continuous inverse.
} 
where the differential mapping is not onto. A regular economy is, by definition, an economy that is not singular. By Sard's theorem since the set $\mathcal{U}$ and the space of economies are two manifolds of same dimension, the set of singular economies is a set of Lebesgue measure zero ${ }^{2}$. By the Implicit Function Theorem, each regular economy has a finite number of equilibria and, around a regular economy, there exist continuously differentiable selections of the equilibrium prices.

Computing the degree of the extended projection by an homotopy argument, we obtain that every regular economy has a finite odd number of equilibrium prices.

We now mention earlier contributions. Rader [25] showed that, when the consumers have demand functions almost everywhere differentiable satisfying property (N): "The image of a null set is a null set.", almost every economy has a finite number of equilibrium prices. In our paper, we prove that Rader's properties are satisfied by multiprior preferences but we get more with the local continuously differentiable selections.

Shannon and Rigotti [26] study market implications of the presence of ambiguity modelled by variational preferences. Variational preferences encompass multiprior preferences. They show that almost all economies are determinate which means that there exist a finite number of equilibrium prices and local continuous selections. They obtain also a Lipschitz behavior in the Choquet case. Note that regularity and determinacy are two distinct concepts, the first one implying the second one. In particular, the number of equilibria may not be constant around a determinate economy. We need the linear independence of Assumption 1 to get regularity instead of determinacy.

In [9], Dana studies agents that are Choquet expected-utility maximizers. She is interested in equilibrium welfare properties and indeterminacy of the equilibrium. She provides a sufficient condition on equilibrium implying that there exists a continuum of equilibrium prices. But, she does not address the issue of genericity.

In [5], Bonnisseau and Rivera-Cayupi study a non-smooth model although the failure of differentiability was not in the utility function but on the boundary of the consumption sets. They obtain demand functions with properties similar to ours.

In Section 2, we present multiprior preferences and the definition of an equilibrium with complete markets. Actually, to simplify the notation, we consider a larger class of preferences where the utility functions are defined as a minimum of a finite family of functions satisfying the usual differentiability requirements and a linear independence assumption on the gradient vectors.

\footnotetext{
${ }^{2}$ Actually, we also use that the image of a null set by a Lipschitz mapping is a null set.
} 
In Section 3, we study extensively the demand function of a consumer with multiprior preferences. The fourth section is devoted to the global analysis of the equilibrium manifold and to the genericity analysis. Some concluding remarks are given in Section 5 and finally, some technical proofs are given in Appendix.

\section{Multiprior preferences}

$\mathrm{We}^{3}$ study a two-period economy with a complete system of markets. There are two dates $t=0$ and $t=1$. There is uncertainty at date 0 about which state will occur at date 1 . At date 1 , there are $S$ states of nature. We denote by $\Delta(S)$ the set of probabilities on $\mathcal{S}=\{1, \ldots, S\}$. There are $I$ goods at each node so there are $\ell:=I(1+S)$ goods. We model the ambiguity by a multiplicity of probabilities.

From a general equilibrium point of view, we study a family of economies parametrized by strictly positive endowments with $m$ consumers and $\ell$ commodities. We denote respectively by $M$ and $L$ the set of consumers and the set of commodities. Let $M \equiv\{1, \ldots, m\}$ and $L \equiv\{1, \ldots, \ell\}$.

For each agent $i \in M$, there exists a closed convex set $\mathcal{P}^{i} \subset \Delta(S)$. We suppose that the set $\mathcal{P}^{i}$ has $n_{i}$ extremal points $\left(\pi_{i}^{k}\right)_{1 \leq k \leq n_{i}}$. We also suppose that the set $\mathcal{P}^{i}$ is contained in $\mathbb{R}_{++}^{S}$ to get the strict monotony of preferences. This can in particular correspond to the convex case of the C.E.U. (Choquet Expected Utility) model of Schmeidler[28] since the core of a convex capacity has at most $S$ ! extremal points (Shapley [29]).

The agent chooses a contingent consumption vector $\left(x_{s}\right)_{1 \leq s \leq S} \in \mathbb{R}_{++}^{I S}$ and a vector $x_{0} \in \mathbb{R}_{++}^{I}$ corresponding to his consumption at date zero. The utility of the agent $i$ is given by:

$$
u_{i}(x)=b_{i 0}\left(x_{0}\right)+\min _{\pi_{i} \in \mathcal{P}^{i}} \sum_{s=1}^{S} \pi_{i}(s) b_{i s}\left(x_{s}\right)=\min _{1 \leq k \leq n_{i}}\left\{b_{i 0}\left(x_{0}\right)+\sum_{s=1}^{S} \pi_{i}^{k}(s) b_{i s}\left(x_{s}\right)\right\}
$$

\footnotetext{
${ }^{3}$ Notations. If $x$ is a vector of $\mathbb{R}^{\ell}$, the norm of the vector $x$ is defined by $\|x\|:=$ $\sum_{h=1}^{\ell}=\left|x_{h}\right|$. The left-derivative of a function defined on an open interval $J \subset \mathbb{R}$ at $t \in J$ is denoted by $\Psi_{l}^{\prime}(t)$. Similarly the right-derivative of $\Psi$ at $t$ is denoted by $\Psi_{r}^{\prime}(t)$. The vector 1 denotes the vector of $\mathbb{R}^{\ell}$ that has all coordinates equal to zero. The inner product of $x$ and $y$ elements of $\mathbb{R}^{\ell}$ is: $x \cdot y:=\sum_{h=1}^{\ell} x_{h} y_{h}$. For all $r>0, B_{o}(a, r)$ (respectively $\left.B_{c}(a, r)\right)$ denotes the open (resp. closed) ball of center $a$ and of radius $r$. $\sharp K$ denotes the cardinal of the set $K$. The vectors are, by convention, column vectors and the transpose of a vector $x$ is denoted by $x^{T}$.
} 
where $b_{i s}: \mathbb{R}_{++}^{I} \longrightarrow \mathbb{R}$ are $1+S$ functions. We define, for $k \in\left\{1, \ldots, n_{i}\right\}$, the function $u_{i}^{k}$ by:

$$
u_{i}^{k}(x)=b_{i 0}\left(x_{0}\right)+\sum_{s=1}^{S} \pi_{i}^{k}(s) b_{i s}\left(x_{s}\right) \text { for } x \in \mathbb{R}_{++}^{(1+I) S} .
$$

In many applications, the function $b_{i s}$ does not depend on the state $s$. The state-dependent case in the expected utility model has been studied by Karni, Schmeidler and Vind[19] and Wakker[30] for example. For more references and a presentation of some applications, see [18].

We posit the following assumption on the probability vectors $\left(\pi_{i}^{k}\right)_{1 \leq k \leq n_{i}}$.

Assumption 1 For every $i \in M$, the probability vectors $\left(\pi_{i}^{k}\right)_{1 \leq k \leq n_{i}}$ are linearly independent.

Note that this assumption holds true when $\mathcal{P}^{i}$ is an $\varepsilon$-contamination of a probability $\bar{\pi}$. Recall that a set $\mathcal{P}$ is called an $\varepsilon$-contamination if: $\mathcal{P}:=$ $(1-\varepsilon)\{\bar{\pi}\}+\varepsilon \Delta(S)$ for some real number $\varepsilon \in] 0 ; 1[$. The extremal points of $\mathcal{P}$ are $(1-\varepsilon) \bar{\pi}+\varepsilon \pi^{s}$ for $s=1, \ldots, S$, where $\pi^{s}$ is the probability such that $\pi^{s}(s)=1$. Obviously, these vectors are linearly independent. The $\varepsilon$ contamination of confidence is a special case of the Choquet Expected Utility model. Indeed, the related capacity $\nu$ is defined by:

$$
\nu(A):=\left\{\begin{array}{l}
(1-\varepsilon) \bar{\pi}(A) \text { if } A \neq \mathcal{S} \\
1 \text { if } A=\mathcal{S}
\end{array}\right.
$$

and the set $\mathcal{P}$ is its core. The weight $1-\varepsilon$ can be interpreted as the degree of confidence the decision maker has in his prior $\bar{\pi}$. This model has been axiomatized by Nishimura and Ozaki[24].

The $\varepsilon$-contamination of confidence has the favor of many economists because it is easier to handle than general multiple prior models. Nishimura and Ozaki[23] applied this model to search behavior, Chen and Epstein[7] to portfolio choice, Epstein and Wang[13] to asset pricing and Carlier, Dana and Shahdi[6] to insurance. For more references, we refer the reader to Nishimura and Ozaki[24] and Kopylov[20].

Let us now present the assumptions on the functions $\left(b_{i s}\right)_{s=0}^{S}$.

Assumption 2 For every $i \in M$, we assume:

1. $\forall s=0, \ldots, S, b_{i s}$ is of class $C^{2}$,

2. $\forall s=0, \ldots, S$ and all $x_{s} \in \mathbb{R}_{++}^{I}, \nabla b_{i s}\left(x_{s}\right) \gg 0$, 
3. $\forall s=0, \ldots, S$ and all $x_{s} \in \mathbb{R}_{++}^{I}, D^{2} b_{i s}\left(x_{s}\right)$ is negative definite.

Assumption 3 For every $i \in M$, for all $s=0, \ldots, S$, if a sequence $\left(x^{\nu}\right)_{\nu \geq 0}$ converges to $\bar{x} \in \mathbb{R}_{+}^{I}$ and $\bar{x}_{h}:=0$ for some $h \in\{1, \ldots, I\}$ then ${ }^{4}$ :

1. $\lim _{\nu \rightarrow+\infty}\left\|\nabla b_{i s}\left(x^{\nu}\right)\right\|=+\infty$,

2.

$$
\lim _{\nu \longmapsto+\infty} \frac{\nabla b_{i s}\left(x^{\nu}\right) \cdot x^{\nu}}{\left\|\nabla b_{i s}\left(x^{\nu}\right)\right\|}=0 .
$$

Remark 2.1 In the classical one-good case, i.e. $I=1$, and in the separable case $^{5}$, this assumption can be replaced by the following Inada condition: If a sequence $\left(x^{\nu}\right)_{\nu \geq 0}$ converges to $\bar{x} \in \mathbb{R}_{+}^{I}$ and $\bar{x}_{h}:=0$ for some $h \in\{1, \ldots, I\}$ then: $\lim _{\nu \rightarrow+\infty} \frac{\partial b_{i s}\left(x^{\nu}\right)}{\partial x_{h}}=+\infty$.

The system of markets is complete. Therefore the budget set can be described by one single constraint with contingent prices at date zero as explained in the last chapter of Debreu[10]. One can also read Chapter 2 of MagillQuinzii[21] for more details about complete and incomplete markets in twoperiod economies. The demand $f_{i}(p, w)$ of consumer $i$ with respect to the price $p:=\left(p_{0},\left(p_{s}\right)_{s=1}^{S}\right) \in \mathbb{R}_{++}^{I(1+S)}$ and to his wealth $w>0$ is the solution of the following problem:

$$
\left\{\begin{array}{l}
\max u_{i}(x) \\
\text { subject to } x \gg 0 \text { and } p \cdot x \leq w
\end{array}\right.
$$

In the next definition, we use the simplex normalization, i.e., we take prices in $\mathbb{P}$ with $\mathbb{P}:=\left\{p \in \mathbb{R}_{++}^{\ell}, \sum_{h=1}^{\ell} p_{h}=1\right\}$. We end this section by the definition of an equilibrium:

Definition 2.1 $p \in \mathbb{P}$ is an equilibrium price of the economy $\mathbf{e}=\left(e_{i}\right)_{i \in M}$ if:

$$
\sum_{i=1}^{m} f_{i s}\left(p, p . e_{i}\right)=\sum_{i=1}^{m} e_{i s} \text { for all } s=0, \ldots, S .
$$

In this paper, since the preferences of the consumers are fixed, we define an economy as an element $\mathbf{e}:=\left(e_{i}\right)_{i=1}^{m}$ of $\left(\mathbb{R}_{++}^{\ell}\right)^{m}$. Therefore, the space of the economies is $\Omega:=\left(\mathbb{R}_{++}^{\ell}\right)^{m}$.

\footnotetext{
${ }^{4}$ Let $v \in \mathbb{R}^{I}$. $\|v\|$ denotes the norm of the vector $v$ defined by $\|v\|:=\sum_{h=1}^{I}\left|v_{h}\right|$.

${ }^{5} \mathrm{~A}$ function $b: \mathbb{R}_{++}^{I} \rightarrow \mathbb{R}$ is said to be separable if there exist $I$ real-valued functions $\left(\rho_{i}\right)_{i=1}^{I}$ defined on $] 0,+\infty\left[\right.$ and $I$ numbers such that: $b(x)=\sum_{i=1}^{I} \beta_{i} \rho_{i}\left(x_{i}\right)$.
} 
The aim of the paper is to prove that regular economies are generic for multiprior preferences. Regular economies exhibit enjoyable properties: odd finite number of equilibrium prices, local constancy of this number, local differentiable selections of the equilibrium prices.

Thus, even if ambiguity aversion is represented by non-differentiable multiprior preferences, economies retain generically the properties of the differentiable approach.

Remark 2.2 Balasko[1] showed that the regular economies are generic in the standard model when the total resources are fixed. This is no more true in our setting. As an example, let us consider the following multiprior model with two identical consumers, two states of the nature and two extremal priors. Formally, $\mathcal{P}^{1}=\mathcal{P}^{2}:=\left[\pi^{1}, \pi^{2}\right]$ where: $\pi^{1}=\left(\frac{1}{2}, \frac{1}{2}\right)$ and $\pi^{2}=\left(\frac{1}{3}, \frac{2}{3}\right)$. The Bernoulli function is $b(x)=\ln (x)$ so the utility function is defined on $\mathbb{R}_{++}^{2}$ by:

$$
u(x):=\min \left\{\frac{1}{2} \ln \left(x_{1}\right)+\frac{1}{2} \ln \left(x_{2}\right) ; \frac{1}{3} \ln \left(x_{1}\right)+\frac{2}{3} \ln \left(x_{2}\right)\right\} .
$$

The demand function defined on $\mathbb{R}_{++}^{3}$ is:

$$
f\left(p_{1}, p_{2}, w\right)=\left\{\begin{array}{l}
\left(\frac{w}{2 p_{1}}, \frac{w}{2 p_{2}}\right) \quad \text { if } p_{2}<p_{1} ; \\
\left.\frac{w}{p_{1}+p_{2}}, \frac{w}{p_{1}+p_{2}}\right) \quad \text { if } p_{1} \leq p_{2} \leq 2 p_{1} \\
\left.\frac{w}{3 p_{1}}, \frac{2 w}{3 p_{2}}\right) \quad \text { if } p_{2}>2 p_{1} .
\end{array}\right.
$$

Suppose that the total resources vector is equal to $r=(1,1)$. The space of the economies with total resources equal to $r$ is denoted by $\Omega(r)$. For every economy $\mathbf{e} \in \Omega(r)$, the set of normalized equilibrium prices is:

$$
\left\{\left(p_{1}, 1-p_{1}\right): p_{1} \in\left[\frac{1}{3}, \frac{1}{2}\right]\right\}
$$

The equilibrium allocations of $\mathbf{e}:=((a, b),(1-a, 1-b))$ with $a$ and $b$ positive are $x_{1}=\left(p_{1} a+\left(1-p_{1}\right) b, p_{1} a+\left(1-p_{1}\right) b\right), x_{2}=\left(p_{1}(1-a)+\left(1-p_{1}\right)(1-\right.$ $b),\left(p_{1}(1-a)+\left(1-p_{1}\right)(1-b)\right)$ for $p_{1} \in\left[\frac{1}{3}, \frac{1}{2}\right]$. So all the economies are singular in $\Omega(r)$.

\section{A larger class of preferences}

To simplify the notations, we consider a larger class of preferences and prove that the aforementioned properties are satisfied for this class of preferences. Namely, we prove the genericity of regular economies and obtain the classical properties of these economies. 
We assume that the preferences of consumer $i \in M$ are represented by a utility function $u_{i}$ from $\mathbb{R}_{++}^{\ell}$ to $\mathbb{R}$ which is the minimum of $n_{i}$ functions:

$$
u_{i}=\min \left\{u_{i}^{1}, u_{i}^{2}, \ldots, u_{i}^{n_{i}}\right\}
$$

with $n_{i} \in \mathbb{N}^{*}$. We state the following assumptions on the utility functions.

Assumption 4 For all $i \in M$, for all $k \in\left\{1, . ., n_{i}\right\}$,

1. $u_{i}^{k}$ is $C^{2}$ on $\mathbb{R}_{++}^{\ell}$,

2. $D^{2} u_{i}^{k}(x)$ is negative definite on $\nabla u_{i}^{k}(x)^{\perp}$ for all $x \in \mathbb{R}_{++}^{\ell}$,

3. $u_{i}^{k}$ satisfy $\nabla u_{i}^{k}(x) \gg 0$ for all $x \in \mathbb{R}_{++}^{\ell}$.

For all $x \in \mathbb{R}_{++}^{\ell}, M_{i}(x)$ denotes the set of the indices of the functions realizing the minimum, i.e. $M_{i}(x):=\left\{k \in\left\{1, \ldots, n_{i}\right\}: u_{i}^{k}(x)=u_{i}(x)\right\}$.

Assumption 5 For all $i \in M$, for all $x \in \mathbb{R}_{++}^{\ell}$, the vectors $\left(\nabla u_{i}^{k}(x)\right)_{k \in M_{i}(x)}$ are linearly independent.

Assumption 6 For all $i \in M$, for all $k \in\left\{1, \ldots, n_{i}\right\}$, if a sequence $\left(x_{i}^{\nu}\right)_{\nu \geq 0}$ converges to $x_{i} \in \partial \mathbb{R}_{++}^{\ell}$ then:

$$
\lim _{\nu \longmapsto+\infty} \frac{\nabla u_{i}^{k}\left(x_{i}^{\nu}\right) \cdot x_{i}^{\nu}}{\left\|\nabla u_{i}^{k}\left(x_{i}^{\nu}\right)\right\|}=0 .
$$

Remark 2.3 Assumption 4 tells us that the preferences are continuous, monotone and strictly convex. Moreover, each commodity is desirable.

For $i \in M$ and $k \in\left\{1, \ldots, n_{i}\right\}$, Assumption 6 on the function $u_{i}^{k}$ is weaker than the usual assumption: for all $x \in \mathbb{R}_{++}^{\ell}$, the closure in $\mathbb{R}^{\ell}$ of the set $\left\{x^{\prime} \in \mathbb{R}_{++}^{\ell} \mid u_{i}^{k}\left(x^{\prime}\right) \geq u_{i}^{k}(x)\right\}$ is contained in $\mathbb{R}_{++}^{\ell}$. The usual closure assumption implies Assumption 6 but both assumptions are not equivalent. For instance, the function $u$ defined on $\mathbb{R}_{++}^{2}$ by: $u\left(x_{1}, x_{2}\right)=\sqrt{x_{1}}+\sqrt{x_{2}}$ satisfies Assumption 6 but not the classical assumption ${ }^{6}$.

The next proposition shows that the multiprior preferences satisfy Assumptions 4, 5 and 6.

Proposition 2.1 Let $i \in M$. Under Assumptions 1, 2 and 3, the corresponding family of functions $\left(u_{i}^{k}\right)_{1 \leq k \leq n_{i}}$ satisfies Assumption 4,5 and 6 .

The proof of this proposition can be found in Appendix.

$$
{ }^{6} \text { Take } \bar{x}:=\left(\frac{1}{4}, \frac{1}{4}\right) \text { and }\left(x^{\nu}:=\left(1+\frac{1}{\nu}, \frac{1}{\nu}\right)\right)_{\nu \geq 1} \text { as a counterexample. }
$$




\section{Properties of the individual demand}

In this section, we study the individual demand $f_{i}(p, w)$ of consumer $i \in M$ defined as the solution of the program:

$$
\left\{\begin{array}{l}
\max u_{i}(x) \\
\text { subject to } x \gg 0 \text { and } p \cdot x \leq w
\end{array}\right.
$$

where $w \in] 0,+\infty\left[\right.$ and $p \in \mathbb{R}_{++}^{\ell}$. Let us present the main result of the section:

Proposition 3.1 Under Assumptions 4, 5 and $6, f_{i}(p, w)$ is a singleton for all $i \in M, p \in \mathbb{R}_{++}^{\ell}$ and $w>0$. The function $f_{i}$ is locally Lipschitz continuous on $\left.\mathbb{R}_{++}^{\ell} \times\right] 0,+\infty\left[\right.$. Furthermore, there exists an open subset $\Omega_{i}^{0}$ in $\left.\mathbb{R}_{++}^{\ell} \times\right] 0,+\infty\left[\right.$ of full Lebesgue measure on which $f_{i}$ is $C^{1}$.

We first sketch the proof. To simplify the notation, we skip the index $i$ during the proof and denote the function $u_{i}^{k}$ by $u^{k}$. In Lemma 6.1, we establish the continuity of $f$ as a consequence of Berge's Theorem. Then, in Lemma 6.3, we obtain that this function is locally Lipschitz continuous thanks to Cornet-Vial[8]. Thanks to Rademacher's Theorem, $f$ is differentiable on a set $\tilde{\Omega}$ that has full Lebesgue measure. Following FiaccoMcCormick[15], we prove in Lemma 6.4 that the function $f$ is continuously differentiable around $(\bar{p}, \bar{w})$ whenever the strict complementarity slackness is satisfied at $(\bar{p}, \bar{w})$ for the optimization problem related to the demand. To conclude, in Lemma 3.1, we show that the function $f$ is not differentiable at $(\bar{p}, \bar{w})$ when the strict complementarity slackness is not satisfied.

More precisely, let us rewrite the optimization problem defining the demand. As explained above, the function $u$ is not differentiable a priori. Therefore Problem 3.1 is not in standard form. Considering this, we rewrite this problem in a standard form where the functions at stake are twice continuously differentiable. To do so, we remark that, for all $\left.(p, w) \in \mathbb{R}_{++}^{\ell} \times\right] 0,+\infty[$, $f(p, w)$ is the solution of the following problem:

$$
\left\{\begin{array}{l}
\min -t \\
\text { subject to } \\
t-u^{k}(x) \leq 0, k=1, \ldots, n \\
p \cdot x-w \leq 0 \\
x \gg 0
\end{array}\right.
$$


The first order conditions related to this problem are the following: there exists $\lambda \in \mathbb{R}_{+}^{n+1}$ such that

$$
\left\{\begin{array}{l}
t-u^{k}(x) \leq 0, \lambda_{k}\left(t-u^{k}(x)\right)=0, k=1, \ldots, n \\
p \cdot x-w \leq 0, \lambda_{n+1}(p \cdot x-w)=0 \\
\sum_{k=1}^{n} \lambda_{k}=1 \\
\lambda_{n+1} p=\sum_{k=1}^{n} \lambda_{k} \nabla u^{k}(x)
\end{array}\right.
$$

Note that this maximization problem is not necessarily convex since the constraint $t-u^{k}(x)$ may not be quasi-convex. Nevertheless, the first order conditions are necessary since the Mangasarian-Fromovitz qualification condition is satisfied and sufficient as shown in Appendix. Furthermore, the linear independence Assumption 5 implies that the multiplier is unique.

Now, let $\Omega^{0}$ defined by:

$$
\Omega^{0}:=\left\{(p, w) \in \mathbb{R}_{++}^{\ell} \times\right] 0 ;+\infty\left[\mid \forall k \in M(f(p, w)), \lambda_{k}(p, w)>0\right\}
$$

We prove that the set $\Omega^{0}$ is an open set on which the function $f$ is continuously differentiable by Lemma 6.4 and that the set of differentiability points of $\tilde{\Omega}$ has full Lebesgue measure thanks to Lemma 6.3 and Rademacher's Theorem. In Lemma 3.1, we show that the function $f$ is not differentiable at any point outside $\Omega^{0}$ which means $\tilde{\Omega}=\Omega^{0}$. Therefore we conclude that $f$ is continuously differentiable on an open set of full Lebesgue measure. Lemma 3.1 constitutes the keystep of the proof and is obtained reasoning by contradiction. We construct a price path $t \mapsto p(t)$ with $p(t)=\bar{p}$ and show that the function $t \mapsto u^{k}[f(p(t), p(t) \cdot f(\bar{p}, \bar{w}))]$ is not differentiable at zero for any $k \in M(f(\bar{p}, \bar{w}))$ such that the corresponding multiplier is equal to zero.

All we have to prove is the following Lemma.

Lemma 3.1 If $(\bar{p}, \bar{w}) \notin \Omega^{0}$ then the function $f$ is not differentiable at $(\bar{p}, \bar{w})$.

Let $(\bar{p}, \bar{w}) \notin \Omega^{0}$. For the remaining of the section, we write: $\bar{x}:=f(\bar{p}, \bar{w})$ and $\bar{\lambda}_{k}:=\lambda_{k}(\bar{p}, \bar{w})$ for $k \in\{1, \ldots, n+1\}$. Since the strict complementarity slackness condition does not hold, $M(f(\bar{p}, \bar{w}))$ can be decomposed the following way: $M(f(\bar{p}, \bar{w}))=K \cup K^{\prime}$ with $K$ and $K^{\prime}$ subsets of $\{1, \ldots, n\}, \bar{\lambda}_{k}>0$ for $k \in K, \bar{\lambda}_{k}=0$ for $k \in K^{\prime}$ and $K^{\prime} \neq \emptyset$. Note that we have: $K \neq \emptyset$ since $\bar{p} \neq 0$ and $\sum_{k \in K} \bar{\lambda}_{k}=1$ since $\sum_{K \cup K^{\prime}} \bar{\lambda}_{k}=1$ and $\bar{\lambda}_{k}=0$ for every $k \in K^{\prime}$.

We define the function $\bar{f}$ on $\mathbb{R}_{++}^{\ell}$ by: $\bar{f}(p):=f(p, p \cdot \bar{x})$. 
In the proof of Lemma 3.1, we use some auxiliary functions that we introduce now. We define a generalized Hicksian demand $\Delta_{K}$ and a demand function $f_{K}$ both related to the functions $\left(u_{k}\right)_{k \in K}$. Based on $f_{K}$, we also define the functions $\bar{f}_{K}$ and $\hat{v}_{K}$.

A generalized Hicksian demand and the related expenditure function:

Let $\bar{v}_{K}=\left(\bar{v}^{k}\right)_{k \in K}:=\left(u^{k}(\bar{x})\right)_{k \in K}$. For $p \in \mathbb{R}_{++}^{\ell}$ and $v_{K}=\left(v^{k}\right)_{k \in K} \in \mathbb{R}^{K}$, let $\Delta_{K}\left(p,\left(v^{k}\right)_{k \in K}\right)$ be the solution ${ }^{7}$ of the problem:

$$
\left\{\begin{array}{l}
\min p \cdot x \\
\text { subject to } u^{k}(x) \geq v^{k} \forall k \in K \\
x \gg 0
\end{array}\right.
$$

The related expenditure function is defined by:

$$
e_{K}\left(p, v_{K}\right):=p \cdot \Delta_{K}\left(p, v_{K}\right)
$$

The map $\Delta_{K}$ has been extensively studied in [4]. This map is continuously differentiable with respect to $(p, v)$ around the point $\left(\bar{p}, \bar{v}_{K}\right)$ since the multipliers related to the satiated constraints are positive and the gradients $\left(\nabla u^{k}(\bar{x})\right)_{k \in K}$ are linearly independent which holds true thanks to Assumption 5 .

Note also that $\Delta_{K}\left(\bar{p}, \bar{v}_{K}\right)=\bar{x}$. Indeed the necessary and sufficient first order conditions are satisfied by $\bar{x}$. Moreover, all the constraints are satiated and have positive related multipliers.

Like the classical expenditure function, the function $e_{K}$ is concave with respect to $p$ so almost everywhere twice differentiable and satisfies $D_{p}^{2} e_{K}=$ $D_{p} \Delta_{K}$ whenever this expression makes sense ${ }^{8}$.

The next lemma is a generalization of a well known result about the Slutsky matrix.

Lemma 3.2 The matrix $D_{p} \Delta_{K}(\bar{p}, \bar{v})$ has rank $\ell-\sharp K$ and its kernel is the linear space $\mathcal{L}\left(\nabla u^{k}(\bar{x}), k \in K\right)$ spanned by the family $\left(\nabla u^{k}(\bar{x})\right)_{k \in K}$.

This result is proved in Appendix.

An auxiliary demand function:

\footnotetext{
${ }^{7}$ In [4], it is proved that this solution exists and is unique for $\left(p, v_{K}\right)$ in a neighborhood of $\left(\bar{p}, \bar{v}_{K}\right)$.

${ }^{8}$ See $[4]$.
} 
We define the demand function $f_{K}$ related to the utility functions $\left(u^{k}\right)_{k \in K}$. $f_{K}(p, w)$ is the solution of the optimization problem:

$$
\left\{\begin{array}{l}
\min -t \\
\text { subject to } \\
t-u^{k}(x) \leq 0 \quad k \in K \\
p \cdot x-w \leq 0 \\
x \gg 0
\end{array}\right.
$$

Note that we have: $f_{K}(\bar{p}, \bar{w})=\bar{x}=f(\bar{p}, \bar{w})$ since the necessary and sufficient first order conditions are satisfied by $\bar{x}$. Indeed, we have: $\bar{\lambda}_{k}=0$ for all $k \in K^{\prime}$. The function $f_{K}$ is continuously differentiable around $(\bar{p}, \bar{w})$ since all the multipliers are positive. See Lemma 6.4.

We define on $\mathbb{R}_{++}^{\ell}$ the function $\bar{f}_{K}$ by: $\bar{f}_{K}(p)=f_{K}(p, p \cdot \bar{x})$. We also define the function $\hat{v}_{K}$ from $\mathbb{R}_{++}^{\ell}$ to $\mathbb{R}^{K}$ by $\hat{v}_{K}(p)=\left(\hat{v}_{K}^{k}(p)=u^{k}\left[\bar{f}_{K}(p)\right]\right)_{k \in K}$. Note that, for $\left(k, k^{\prime}\right) \in K^{2}, \hat{v}_{K}^{k}(p)=\hat{v}_{K}^{k^{\prime}}(p)$ in a neighborhood of $\bar{p}$. Indeed, from the necessary and sufficient first order conditions, $\bar{f}_{K}(p)=\Delta_{K}\left(p, \hat{v}_{K}(p)\right)$ for $p$ close enough to $\bar{p}$ because the function $\hat{v}_{K}$ and the multipliers corresponding to Problem 3.5 are continuous ${ }^{9}$ and the multipliers are all positive at $\left(\bar{p}, \bar{v}_{K}\right)$. To conclude, we point out the following facts:

- $\bar{x}=\Delta_{K}\left(\bar{p}, \bar{v}_{K}\right)=f_{K}(\bar{p}, \bar{w})$,

- for $p$ close enough to $\bar{p}$, we have: $\bar{f}_{K}(p)=\Delta_{K}\left(p, \hat{v}_{K}(p)\right)$,

- The functions $f_{K}, \bar{f}_{K}, \Delta_{K}$ and $\hat{v}_{K}$ are continuously differentiable respectively locally around $(\bar{p}, \bar{w}), \bar{p},(\bar{p}, \bar{v})$ and $\bar{p}$.

The following result relates the partial derivative with respect to $p$ of the compensated demand $\Delta_{K}$ and the differential of the demand $\bar{f}_{K}$ at the price $\bar{p}$ and provides an important result concerning the functions $\left(\hat{v}_{K}^{k}\right)_{k \in K}$.

Lemma 3.3 For all $k \in K, \nabla \hat{v}_{K}^{k}(\bar{p})=0$. Moreover we have:

$$
D \bar{f}_{K}(\bar{p})=D_{p} \Delta_{K}\left(\bar{p}, \bar{v}_{K}\right)
$$

The proof is given in Appendix.

\section{Proof of Lemma 3.1}

Recall that we have: $M(f(\bar{p}, \bar{w}))=K \cup K^{\prime}$ with $K$ and $K^{\prime}$ subsets of $\{1, \ldots, n\}$ and that $\bar{\lambda}_{k}>0$ for $k \in K$ and $\bar{\lambda}_{k}=0$ for $k \in K^{\prime}$.

We now consider a particular price path $p($.$) to get the desired result.$ Let $E$ be the vector space spanned by the family $\left(\nabla u^{k}(\bar{x})\right)_{k \in K^{\prime}}$ and $\Phi:=$

\footnotetext{
${ }^{9}$ This is a consequence of Lemma 6.3.
} 
$D_{p} \Delta_{K}(\bar{p}, \bar{v})$. From Lemma 3.2, the matrix $D_{p} \Delta_{K}(\bar{p}, \bar{v})$ is negative semidefinite on $\mathbb{R}^{\ell}$ and $\operatorname{Ker} D_{p} \Delta_{K}(\bar{p}, \bar{v})=\mathcal{L}\left(\nabla u^{k}(\bar{x}), k \in K\right)$. On the other hand, the family $\left(\nabla u^{k}(\bar{x})\right)_{k \in K \cup K^{\prime}}$ is linearly independent from Assumption 5 . Hence $D_{p} \Delta_{K}(\bar{p}, \bar{v})$ is negative definite on $E$ since $\operatorname{Ker} D_{p} \Delta_{K}(\bar{p}, \bar{v}) \cap E=\{0\}$.

Thanks to Proposition 6.1, there exists $\left(\alpha_{k}\right)_{k \in K^{\prime}}$ in $\mathbb{R}_{++}^{K^{\prime}}$ such that, for all $k^{\prime} \in K^{\prime}$,

$$
\nabla u^{k^{\prime}}(\bar{x}) \cdot D_{p} \Delta_{K}(\bar{p}, \bar{v})\left(\sum_{k \in K^{\prime}} \alpha_{k} \nabla u^{k}(\bar{x})\right)<0 .
$$

For all $t \in J:=]-a, a\left[^{10}\right.$, let :

$$
p(t):=\frac{1}{\bar{\lambda}_{n+1}}\left[\sum_{k \in K} \bar{\lambda}_{k} \nabla u^{k}(\bar{x})+t\left(\sum_{k \in K^{\prime}} \alpha_{k} \nabla u^{k}(\bar{x})\right)\right] .
$$

For $k \in K$, we introduce the function defined on $J$ by: $\tilde{v}^{k}(t):=\hat{v}^{k}(p(t))=$ $u^{k}\left[\bar{f}_{K}(p(t))\right]$. For $k \in K^{\prime}$, we define the functions $\varphi^{k}$ and $\tilde{\varphi}^{k}$ on $J$ by: $\varphi^{k}(t):=$ $u^{k}[\bar{f}(p(t))]$ and $\tilde{\varphi}^{k}(t):=u^{k}\left[\bar{f}_{K}(p(t))\right]^{11}$. As a preliminary remark, we see that the functions $\left(\tilde{\varphi}^{k}\right)_{k \in K^{\prime}}$ and $\left(\tilde{v}_{k}\right)_{k \in K}$ are differentiable on a neighborhood of zero. Indeed, the functions $\left(u^{k}\right)_{k=1}^{n}$ are differentiable, the function $\bar{f}_{K}$ is differentiable around $\bar{p}, p(0)=\bar{p}$ and $p($.$) is a differentiable path on J$.

Claim 3.1 If $t>0$ is small enough, $\bar{f}(p(t))=f(p(t), p(t) \cdot \bar{x})=\bar{x}$ so the right-derivative $\left(\varphi^{k}\right)^{\prime r}(0)$ is equal to zero for all $k \in K^{\prime}$.

Proof of Claim 3.1 For $t>0$ small enough, thanks to the formula 3.7, one remarks that $\bar{x}$ satisfies the necessary and sufficient first order conditions related to the problem:

$$
\left\{\begin{array}{l}
\max u(x) \\
\text { s.t. } x \gg 0 \\
p(t) \cdot x \leq p(t) \cdot \bar{x}
\end{array}\right.
$$

with the related multipliers

$$
\frac{\bar{\lambda}_{k}}{\sum_{\kappa \in K} \bar{\lambda}_{\kappa}+t\left(\sum_{\kappa \in K^{\prime}} \alpha_{\kappa}\right)} \text { for } k \in K, \frac{t \alpha_{k}}{\sum_{\kappa \in K} \bar{\lambda}_{\kappa}+t\left(\sum_{\kappa \in K^{\prime}} \alpha_{\kappa}\right)} \text { for } k \in K^{\prime} \text {, }
$$

\footnotetext{
${ }^{10}$ The number $a$ is a positive number sufficiently small to ensure that $p(t)$ belongs to $\mathbb{R}_{++}^{\ell}$ for all $t \in J$.

${ }^{11}$ To avoid any confusion, we denote with " $\varphi$ "s the functions for $k \in K^{\prime}$ and with " $v$ "s the functions for $k \in K$.
} 
0 for $k \notin M(\bar{x}) \cup\{n+1\}$ and $\frac{\bar{\lambda}_{n+1}}{\sum_{\kappa \in K} \bar{\lambda}_{\kappa}+t\left(\sum_{\kappa \in K^{\prime}} \alpha_{\kappa}\right)}$ for the budget constraint $^{12}$. Therefore we can conclude that $\bar{x}$ is a solution of the problem, that is: $f(p(t), p(t) \cdot \bar{x})=\bar{f}(p(t))=\bar{x}$.

From Lemma 3.3 and the choice of the family $\left(\alpha_{k}\right)_{k \in K^{\prime}}$, we obtain the following claim:

Claim 3.2 For all $k \in K^{\prime}$, we have:

$$
\left(\tilde{\varphi^{k}}\right)^{\prime}(0)<0 .
$$

Proof of Claim 3.2 By the chain rule, Lemma 3.3 and Equation (3.6), we have for $k \in K^{\prime}$ :

$$
\begin{aligned}
\left(\tilde{\varphi^{k}}\right)^{\prime}(0) & =\nabla u^{k}(\bar{x}) \cdot D \bar{f}_{K}(\bar{p})\left(\sum_{\kappa \in K^{\prime}} \alpha_{\kappa} \nabla u^{\kappa}(\bar{x})\right) \\
& =\nabla u^{k}(\bar{x}) \cdot D_{p} \Delta_{K}\left(\bar{p}, \bar{v}_{K}\right)\left(\sum_{\kappa \in K^{\prime}} \alpha_{\kappa} \nabla u^{\kappa}(\bar{x})\right) \\
& <0
\end{aligned}
$$

Now we consider the last claim.

Claim $3.3 \bar{f}_{K}(p(t))=\bar{f}(p(t))$ for $t<0$ sufficiently near from zero. Consequently, the left-derivative $\left(\varphi^{k}\right)^{l}(0)$ is negative for every $k \in K^{\prime}$.

Proof of Claim 3.3 We first say that the constraints corresponding to $k \in$ $\{1, \ldots, n\} \backslash M(f(p, w))$ are inactive for $t<0$ sufficiently small by continuity of the functions at stake. Summarizing what we obtained before, we have:

- $\left(\tilde{v}^{k}\right)^{\prime}(0)=0$ for $k \in K($ Lemma 3.3),

- $\left(\tilde{\varphi}^{k}\right)^{\prime}(0)<0$ for $k \in K^{\prime}($ Claim 3.2),

- $\tilde{v}^{k}(0)=\left(\tilde{\varphi}^{k}\right)^{\prime}(0)$ for $\left(k, k^{\prime}\right) \in K \times K^{\prime}$.

The last equality comes from the following equalities:

$$
p(0)=\bar{p}, f_{K}(\bar{p}, \bar{w})=f_{K}(\bar{p}, \bar{p} \cdot \bar{x})=f(\bar{p}, \bar{w})=\bar{x}
$$

and $u^{k}(\bar{x})=u^{k^{\prime}}(\bar{x})$ for all $\left(k, k^{\prime}\right) \in K \times K^{\prime}$.

From these three facts, we get: $\left(\tilde{\varphi}_{k}\right)^{\prime}(t)>\hat{v}^{k}(t)$ for all $\left(k, k^{\prime}\right) \in K \times K^{\prime}$ and $t<0$ sufficiently near from zero. Therefore $\bar{f}_{K}(p(t))$ is feasible for $t$ close enough to zero for Problem 3.2 defining the demand $f(p(t), p(t) \cdot \bar{x})$. It satisfies the necessary and sufficient first order conditions and the multipliers

\footnotetext{
12 See Equation 6.3.
} 
are given by the multipliers related to Problem 3.5 completed by zeroes for $k \in K^{\prime}$ and $k \notin M[f(\bar{p}, \bar{w})]$. Since $\overline{f_{K}}(p(t))=\bar{f}(p(t))$ for $t<0$ close enough to zero, we have $\varphi^{k}(t)=\tilde{\varphi}^{k}(t)$ for $t<0$ close enough to zero for all $k \in K^{\prime}$. We conclude: $\left(\varphi^{k}\right)^{\prime l}(0)<0$ for every $k \in K^{\prime}$ by Claim 3.2.

Finally, for every $k \in K^{\prime}$, from Claim 3.3, the left derivative of $\varphi^{k}$ at 0 is negative while the right derivative of $\varphi^{k}$ at 0 is equal to zero by Claim 3.1. Therefore the functions $\left(\varphi^{k}\right)_{k \in K^{\prime}}$ are not differentiable at zero, which implies that the function $f$ is not differentiable at $(\bar{p}, \bar{w})$.

\section{The equilibrium manifold}

In this section, we study the equilibrium price vectors from a global point of view following Balasko [1]. The monotony of the utility functions implies that the equilibrium prices are always strictly positive. Moreover they are defined up to a normalization. We use the simplex normalization, i.e., we take prices in $\mathbb{S}$ with $\mathbb{S}:=\left\{p \in \mathbb{R}_{++}^{\ell}, \sum_{h=1}^{\ell} p_{h}=1\right\}$. We now define the equilibrium manifold and the natural projection.

Definition 4.1 (i) $p \in \mathbb{S}$ is an equilibrium price of the economy $\mathbf{e}=\left(e_{i}\right)_{i \in M}$ if:

$$
\sum_{i=1}^{m} f_{i}\left(p, p . e_{i}\right)=\sum_{i=1}^{m} e_{i} .
$$

(ii) The equilibrium manifold $E_{e q}$ is the set of the pairs $(p, \mathbf{e}) \in \mathbb{S} \times \Omega$ with $p$ equilibrium price for the economy e. An element $(p, \mathbf{e})$ of the set $E_{\text {eq }}$ is called an equilibrium point.

In our framework, the equilibrium manifold ${ }^{13}$ is not necessarily smooth and we cannot directly apply the classical arguments of differential topology. Nevertheless, we parametrize the equilibrium manifold and exploit the results of the previous section.

We recall that $\mathbf{1}$ denotes the vector whose coordinates are all equal to 1 and by $\mathbf{1}^{\perp}$ the vector space orthogonal to the vector $\mathbf{1}$. We define the mapping $\theta^{2}$ from $\mathcal{X}=\mathbb{S} \times \mathbb{R}_{++}^{m} \times\left(\mathbf{1}^{\perp}\right)^{m-1}$ to $\left(\mathbb{R}^{\ell}\right)^{m}$ as follows: for $\xi=$ $\left(p,\left(w_{i}\right)_{i=1}^{m},\left(\eta_{i}\right)_{i=1}^{m-1}\right)$,

$$
\left\{\begin{array}{c}
\theta_{i}^{2}(\xi):=\eta_{i}+\left(w_{i}-p \cdot \eta_{i}\right) \mathbf{1} \forall i=1, \ldots, m-1 \\
\theta_{m}^{2}(\xi):=f_{m}\left(p, w_{m}\right)+\sum_{i=1}^{m-1}\left(f_{i}\left(p, w_{i}\right)-\theta_{i}^{2}(\xi)\right)
\end{array}\right.
$$

\footnotetext{
${ }^{13}$ We prove that it is indeed a smooth manifold at almost every point.
} 
Let us define the set $\mathcal{U}$ by

$$
\mathcal{U}:=\left\{\xi \in \mathcal{X} \mid \theta^{2}(\xi) \in\left(\mathbb{R}_{++}^{\ell}\right)^{m}\right\} .
$$

Now we define the subset $\mathcal{V}$ of $\mathcal{U}$, as follows: an element $\left(p,\left(w_{i}\right)_{i=1}^{m},\left(\eta_{i}\right)_{i=1}^{m-1}\right)$ of $\mathcal{U}$ belongs to $\mathcal{V}$ if and only if $\left(p, w_{i}\right)$ belongs to $\Omega_{i}^{0}$ for all $i=1, \ldots, m, \Omega_{i}^{0}$ being given by Proposition 3.1.

Proposition 4.1 The set $\mathcal{U}$ is an open connected subset of $\mathbb{S} \times \mathbb{R}^{m} \times\left(\mathbf{1}^{\perp}\right)^{m-1}$.

Proof The set $\mathcal{U}$ is clearly open in $\mathbb{S} \times \mathbb{R}^{m} \times\left(\mathbf{1}^{\perp}\right)^{m-1}$ as $\theta^{2}$ is continuous. For the remaining of the proof, we will use extensively the following result. For all $x \in \mathbb{R}^{\ell}$ and all $p \in \mathbb{S}$, we have:

$$
x=\operatorname{proj}_{\mathbf{1}^{\perp}} x+\left(p \cdot x-p \cdot \operatorname{proj}_{\mathbf{1}^{\perp}} x\right) \mathbf{1} .
$$

We show that the set $\mathcal{U}$ is arcconnected. Let $\xi^{k}:=\left(p^{k},\left(w_{i}^{k}\right)_{i=1}^{m},\left(\eta_{i}^{k}\right)_{i=1}^{m-1}\right)$, $k=1,2$, two elements of $\mathcal{U}$. Our goal is to connect $\xi^{1}$ to $\xi^{2}$. We introduce two intermediate points: $\chi^{k}:=\left(p^{k},\left(w_{i}^{k}\right)_{i=1}^{m},\left(\operatorname{proj}_{1^{\perp}} f_{i}\left(p^{k}, w_{i}^{k}\right)\right)_{i=1}^{m-1}\right), k=1,2$.

We show that we can construct a continuous path between $\xi^{k}$ and $\chi^{k}$, $k=1,2$ and another one between $\chi^{1}$ and $\chi^{2}$, theses paths taking values in $\mathcal{U}$ which gives us the result. We first remark that Formula 4.2 implies that $\theta^{2}\left(\chi^{k}\right)=\left(f_{i}\left(p^{k}, w_{i}^{k}\right)\right)_{i=1}^{m}$ for $k=1,2$.

Paths between $\xi^{k}$ and $\chi^{k}$

For $k=1,2$, for all $t \in[0,1]$, let $\zeta^{k t}:=(1-t) \xi^{k}+t \chi^{k}$. This defines a continuous path between $\xi^{k}$ and $\chi^{k}$. $\zeta^{k t}$ belongs to $\mathcal{U}$ for all $t$ since $\theta^{2}$ is linear with respect to the variables $\left(\eta_{i}\right)_{i=1}^{m-1}$ and $\left(\mathbb{R}_{++}^{\ell}\right)^{m}$ is convex.

Path between $\chi^{1}$ and $\chi^{2}$

For all $t \in[0,1]$, we define $p^{t}:=(1-t) p^{1}+t p^{2}$ and $w_{i}^{t}:=(1-t) w_{i}^{1}+t w_{i}^{2}$ for all $i=1, \ldots, m$. The vector $\chi^{t+1}:=\left(p^{t},\left(w_{i}^{t}\right)_{i=1}^{m},\left(\operatorname{proj}_{1^{\perp}} f_{i}\left(p^{t}, w_{i}^{t}\right)\right)_{i=1}^{m-1}\right)$ defines a continuous path between $\chi^{1}$ and $\chi^{2}$ thanks to the continuity of the demand functions and belongs to $\mathcal{U}$ since $\theta^{2}\left(\chi^{t+1}\right)=\left(f_{i}\left(p^{t}, w_{i}^{t}\right)\right)_{i=1}^{m} \in$ $\left(\mathbb{R}_{++}^{\ell}\right)^{m}$.

We conclude that the set $\mathcal{U}$ is arcconnected

Now, we introduce the map $\theta$ from $\mathcal{U}$ to $E_{\text {eq }}$ to parametrize the equilibrium manifold. For $\xi=\left(p,\left(w_{i}\right)_{i=1}^{m},\left(\eta_{i}\right)_{i=1}^{m-1}\right) \in \mathcal{U}$,

$$
\theta(\xi)=\left(p, \theta^{2}(\xi)\right) \text {. }
$$

Note that the range of the map $\theta$ is contained in $E_{e q}$. Let $e_{i}=\theta_{i}^{2}(\xi)$. From the formula defining $\theta^{2}$, one has $p \cdot e_{i}=w_{i}$, from the definition of $\mathcal{U}$, $e_{i} \gg 0$ for all $i$ in $M$ and from the formula for $\theta_{m}^{2}, \sum_{i=1}^{m} f_{i}\left(p, p \cdot e_{i}\right)=\sum_{i=1}^{m} e_{i}$. 
The other map that we consider is the following:

$$
\begin{aligned}
\phi: E_{e q} & \longrightarrow \mathcal{U} \\
\left(p,\left(e_{i}\right)_{i=1}^{m}\right) & \longmapsto\left(p,\left(p . e_{i}\right)_{i=1}^{m},\left(\operatorname{proj}_{1^{\perp}} e_{i}\right)_{i=1}^{m-1}\right) .
\end{aligned}
$$

Note that the range of the map $\phi$ is contained in $\mathcal{U}$. Indeed, from Formula 4.2, $\theta^{2}\left(p,\left(p \cdot e_{i}\right)_{i=1}^{m},\left(\operatorname{proj}_{1^{\perp}} e_{i}\right)_{i=1}^{m-1}\right)=\left(e_{i}\right)_{i=1}^{m}$ belongs to $\left(\mathbb{R}_{++}^{\ell}\right)^{m}$.

We now state the properties of $\theta$ and $\phi$, which imply that $E_{e q}$ is a manifold parametrized by $\theta$.

\section{Proposition 4.2}

1. The maps $\theta$ and $\phi$ are one-to-one, onto and $\theta^{-1}=\phi$.

2. The maps $\theta$ and $\phi$ are locally Lipschitz and continuous.

3. The set $\mathcal{U} \backslash \mathcal{V}$ is closed in $\mathcal{U}$ and has Lebesgue measure zero.

4. $\theta$ is continuously differentiable on $\mathcal{V}$.

5. $E_{\text {eq }}$ is lipeomorphic to $\mathcal{U}$.

Proof The proof is based on the properties of the demand functions.

1. As already noticed above, from Formula 4.2 , for all $\left(p,\left(e_{i}\right)_{i=1}^{m}\right) \in E_{e q}$, $\theta \circ \phi\left(p,\left(e_{i}\right)_{i=1}^{m}\right)=\left(p,\left(e_{i}\right)_{i=1}^{m}\right)$.

Conversely, for all $\xi=\left(p,\left(w_{i}\right)_{i=1}^{m},\left(\eta_{i}\right)_{i=1}^{m-1}\right) \in \mathcal{U}$, the definition of $\theta^{2}$ and Walras law imply that $p \cdot \theta_{i}^{2}(\xi)=w_{i}$ for $i=1, \ldots, m$, and $\operatorname{proj}_{\mathbf{1}^{\perp}} \theta_{i}^{2}(\xi)=\eta_{i}$ for $i=1, \ldots, m-1$. So, $\phi \circ \theta(\xi)=\xi$.

Hence, $\phi=\theta^{-1}$ and $\phi$ and $\theta$ are one-to-one and onto.

2. $\theta$ and $\phi$ are locally Lipschitz and continuous since the demand functions $f_{i}$ are so.

3. Since the set $(\mathbb{S} \times \mathbb{R}) \backslash \Omega_{i}^{0} \cap(\mathbb{S} \times \mathbb{R})$ is a closed set of Lebesgue measure zero for each $i \in M$, the set $\mathcal{U} \backslash \mathcal{V}$ is closed in $\mathcal{U}$ and has Lebesgue measure zero from Fubini's Theorem.

4. The map $\theta$ is $C^{1}$ on $\mathcal{V}$ from the definition of $\mathcal{V}$ and the properties of the demand function $f_{i}$ on $\Omega_{i}^{0}$.

5. This is a consequence of 1 . and 2 .

Following Balasko [1], let us introduce the natural projection as well as the extended projection. 
Definition 4.2 (The natural projection) The natural projection $\pi$ is the map from $E_{e q}$ to $\Omega$ defined by:

$$
\pi: \begin{aligned}
E_{e q} & \longrightarrow \Omega \\
(p, \mathbf{e}) & \longmapsto \mathbf{e}
\end{aligned}
$$

The map $\Pi:=\pi \circ \theta$ is called the extended projection.

$\Pi: \mathcal{U} \longrightarrow \Omega$ is defined by: $\Pi\left(p,\left(w_{i}\right)_{i=1}^{m},\left(\eta_{i}\right)_{i=1}^{m-1}\right):=\theta^{2}\left(p,\left(w_{i}\right)_{i=1}^{m},\left(\eta_{i}\right)_{i=1}^{m-1}\right)$.

Proposition 4.3 The mapping $\Pi$ is proper, locally Lipschitz continuous. Moreover the mapping $\Pi$ is continuously differentiable on $\mathcal{V} . \Pi(\mathcal{U} \backslash \mathcal{V})$ is closed and has Lebesgue measure zero in $\Omega$.

Proof The mapping $\Pi$ is locally Lipschitz continuous and continuously differentiable on the set $\mathcal{V}$ by the properties of $\theta$. The properness of $\Pi$ is a particular case of the properness of $F$, the proof of which is given below as part of the proof of Theorem 4.3.

The set $\Pi(\mathcal{U} \backslash \mathcal{V})$ is closed in $\Omega$ because the set $\mathcal{U} \backslash \mathcal{V}$ is closed in $\mathcal{U}$ and the map $\Pi$ is proper on $\mathcal{U}$. The set $\Pi(\mathcal{U} \backslash \mathcal{V})$ has Lebesgue measure zero because the map $\Pi$ is locally Lipschitz continuous ${ }^{14}$ and the set $\mathcal{U} \backslash \mathcal{V}$ is a null set.

There are different concepts of regularity for non-smooth mappings. In this paper, a regular point is a point where the mapping is differentiable and the differential mapping is onto. A value is regular if all pre-images are regular points. A value is singular if it is not regular.

Definition 4.3 The economy $\mathbf{e}:=\left(e_{i}\right)_{i \in M}$ is called regular if $\mathbf{e} \notin \Pi(\mathcal{U} \backslash \mathcal{V})$ and if the differential of $\Pi$ at all the pre-images of $\mathbf{e}$ is onto. An economy which is not regular is called singular. $E^{r}$ denotes the set of regular economies and $E^{s}$ the set of singular economies.

The following result is the extension of one of the cornerstones of the differentiable approach of general equilibrium theory.

Theorem 4.1 The set of regular economies $E^{r}$ is an open dense subset of $\Omega$ of full Lebesgue measure.

Proof $E^{r}$ is open and has full Lebesgue measure. We have already seen that $\Pi(\mathcal{U} \backslash \mathcal{V})$ is a closed null set. The set of the critical points of $\Pi_{\mid \mathcal{V}}$ is closed in $\mathcal{V}$, hence this set has the form $\mathcal{V} \cap C$ where $C$ is a set closed in $\mathcal{U}$. Remark

\footnotetext{
${ }^{14}$ The image of a null set by a locally Lipschitz map is a null set. See Federer [14].
} 
that we have the equalities: $E^{s}=\Pi(C \cap \mathcal{V}) \cup \Pi(\mathcal{U} \backslash \mathcal{V})=\Pi(C) \cup \Pi(\mathcal{U} \backslash \mathcal{V})$. We deduce that the set $E^{s}$ is a closed set since the map $\Pi$ is proper. This set has Lebesgue measure zero by Sard's Theorem and the previous theorem. Hence, $E^{r}$ is an open set of full Lebesgue measure.

$E^{r}$ is dense. Indeed, the set $E^{s}$ is a set of Lebesgue measure zero, so its complement $E^{r}$ is dense.

The following result summarizes the properties of regular economies. It is a direct consequence of the Implicit Function Theorem.

\section{Theorem 4.2}

1. For $\mathbf{e} \in E^{r}$, there exists a finite number $n$ of equilibrium prices.

2. For $\mathbf{e} \in E^{r}$, there exists an open neighborhood $U \subset E^{r}$ of $\mathbf{e}$ such that the inverse image of $U$ is the union of a finite number of pairwise disjoint subsets $\left(V_{k}\right)_{k=1}^{n}$ of $\mathcal{V}$ and such that the restriction of $\Pi$ to $V_{k}$ is a diffeomorphism for all $k=1, \ldots, n$.

3. For $\mathbf{e} \in E^{r}$, there exist an open neighborhood $U \subset E^{r}$ of $\mathbf{e}$ and a finite number $n$ of continuously differentiable maps $s_{k}: U \longrightarrow \mathbb{S}$ such that the union $\cup_{k=1}^{n} s_{k}\left(\mathbf{e}^{\prime}\right)$ is the set of equilibrium price vectors of the economy $\mathbf{e}^{\prime} \in U$.

Remark 4.1 Note that, around a regular economy e, the number of equilibrium prices is constant by the previous theorem.

We now turn ourselves to the computation of the degree of $\Pi$. We first remark that the mapping $\Pi$ is not continuously differentiable. So we cannot use the classical definition of the degree. Therefore we consider the degree for continuous mappings ${ }^{15}$. Since the set $\mathcal{U}$ is unbounded, the definition of the degree needs some properness assumption ${ }^{16}$.

\section{Theorem 4.3}

1. The map $\Pi$ is of degree one and onto.

2. For all $\mathbf{e} \in \Omega$, there exists an equilibrium.

3. For all $\mathbf{e} \in E^{r}$, there exists a finite odd number of equilibrium prices.

\footnotetext{
${ }^{15} \mathrm{~A}$ good reference is Deimling [12] for example.

${ }^{16}$ See Deimling [12] p.27.
} 
Proof We consider the extended projection $\Pi^{1}$ corresponding to consumers having as utility functions the functions $u_{i}^{1} \cdot{ }^{17}$

The map ${ }^{18} F$ from $\mathbb{S} \times \mathbb{R}_{++}^{m} \times\left(\mathbf{1}^{\perp}\right)^{m-1} \times[0,1]$ to $\mathbb{R}^{\ell m}$ is defined for all $(\xi, t)=\left(\left(p,\left(w_{i}\right)_{i=1}^{m},\left(\eta_{i}\right)_{i=1}^{m-1}\right), t\right) \in \mathbb{S} \times \mathbb{R}_{++}^{m} \times\left(\mathbf{1}^{\perp}\right)^{m-1} \times[0,1]$ by:

$$
F(\xi, t):=t \Pi(\xi)+(1-t) \Pi^{1}(\xi) .
$$

We first show that the inverse image of every compact subset $\mathcal{K}$ of $\Omega$ is a compact subset of $\mathbb{S} \times \mathbb{R}_{++}^{m} \times\left(\mathbf{1}^{\perp}\right)^{m-1} \times[0,1]$. Let us consider a sequence $\left(\xi^{\nu}:=\left(p^{\nu},\left(w_{i}^{\nu}\right)_{i=1}^{m},\left(\eta_{i}^{\nu}\right)_{i=1}^{m-1}\right), t^{\nu}\right)_{\nu \geq 0}$ of $F^{-1}(\mathcal{K})$. We denote by $\left(\mathbf{e}^{\nu}\right)_{\nu \geq 0}$ the sequence of $\mathcal{K}$ defined by $\mathbf{e}^{\nu}:=F\left(\xi^{\nu}, t^{\nu}\right)$ for $\nu \geq 0$. The sequence $\left(\xi^{\nu}, t^{\nu}\right)_{\nu \geq 0}$ remains in a compact set of $\overline{\mathbb{S}} \times \mathbb{R}^{m} \times\left(\mathbf{1}^{\perp}\right)^{m-1} \times[0,1]$. Indeed, the first and the last components lie in a compact set by definition. Moreover, the set $\mathbb{A}:=\left\{\left(p \cdot e_{i}\right)_{i=1}^{m} \mid\left(e_{i}\right)_{i=1}^{m} \in \mathcal{K}, p \in \overline{\mathbb{S}}\right\}$ is a compact set. Since $p \in \overline{\mathbb{S}}$ and since the set $\mathcal{K}$ is contained in $\Omega$, every element $a \in \mathbb{A}$ is positive i.e. $a \in \mathbb{R}_{++}^{m}$. By Walras law and the definition of $F, w_{i}^{\nu}=p^{\nu} \cdot e_{i}^{\nu}$ for all $i=1, \ldots, m$ and all $\nu \geq 0$. Hence, the sequence $\left(\left(w_{i}^{\nu}\right)_{i=1}^{m}\right)_{\nu \geq 0}$ lies in the compact set $\mathbb{A}$.

The compactness of the set $\mathcal{K}$ and the continuity of the projection map imply that the sequence $\left(\eta_{i}^{\nu}:=\operatorname{proj}_{1^{\perp}} e_{i}^{\nu}\right)_{\nu \geq 0}$ lies in a compact subset of $\mathbf{1}^{\perp}$. To conclude, up to a subsequence, the sequence $\left(\xi^{\nu}, t^{\nu}\right)_{\nu \geq 0}$ converges to a vector $\left(\bar{\xi}:=\left(\bar{p},\left(\bar{w}_{i}\right)_{i=1}^{m},\left(\bar{\eta}_{i}\right)_{i=1}^{m-1}\right), \bar{t}\right) \in \overline{\mathbb{S}} \times \mathbb{R}^{m} \times\left(\mathbf{1}^{\perp}\right)^{m-1} \times[0,1]$. Remark that $\left(\bar{w}_{i}\right)_{i=1}^{m}$ belongs to $\mathbb{A}$ so the real number $\bar{w}_{i}$ is positive for all $i=1, \ldots, m$.

Now, we have to prove that this vector belongs to $F^{-1}(\mathcal{K})$. Thanks to the continuity of $F$ and to the closedness of $\mathcal{K}$, it suffices to prove that the price $\bar{p}$ belongs to $\mathbb{S}$. By definition of $F, \Pi$ and $\Pi^{1}$, the $m$-th component of $F, F_{m}\left(\xi^{\nu}, t^{\nu}\right)$ is equal to $\left(1-t^{\nu}\right)\left[f_{m}^{1}\left(p^{\nu}, w_{m}^{\nu}\right)+\sum_{i=1}^{m-1}\left(f_{i}^{1}\left(p^{\nu}, w_{i}^{\nu}\right)-e_{i}^{\nu}\right)\right]+$ $t^{\nu}\left[f_{m}\left(p^{\nu}, w_{m}^{\nu}\right)+\sum_{i=1}^{m-1}\left(f_{i}\left(p^{\nu}, w_{i}^{\nu}\right)-e_{i}^{\nu}\right)\right]$ for $\nu \geq 0$.

We first remark that, for all $i=1, \ldots, m,\left(e_{i}^{\nu}\right)_{\nu \geq 0}$ is bounded since $\left(\mathbf{e}^{\nu}\right)_{\nu \geq 0}$ belongs to the compact set $\mathcal{K}$. We also remark that $f_{i}\left(p^{\nu}, w_{i}^{\nu}\right)$ and $f_{i}^{1}\left(p^{\nu}, w_{i}^{\nu}\right)$ are positive. If $\left(p^{\nu}\right)_{\nu \geq 0}$ converged to $\bar{p}$ in $\partial \mathbb{S}$ and $\left(w_{i}^{\nu}\right)_{i \in M, \nu \geq 0}$ converged to some element $\left(\bar{w}_{i}\right)_{i \in M} \in \mathbb{R}_{++}^{m}$ then $\left\|f_{i}^{1}\left(p^{\nu}, w_{i}^{\nu}\right)\right\|$ and $\left\|f_{i}\left(p^{\nu}, w_{i}^{\nu}\right)\right\|$ would go to $+\infty$ as $\nu$ goes to infinity for all $i \in M$ by monotony of the functions $\left(u_{i}\right)_{i \in M}$ and $\left(u_{i}^{1}\right)_{i \in M}$. So $\left\|F_{m}\left(\xi^{\nu}, t^{\nu}\right)\right\|$ would go to $+\infty$, which contradicts that $F\left(\xi^{\nu}, t^{\nu}\right)$ belongs to the compact set $\mathcal{K}$ for all $\nu \geq 0$.

\footnotetext{
${ }^{17}$ As it can be easily understood, the definitions of $\Pi^{1}, f_{i}^{1}$ and $\theta^{1}$ are analogous to those of $\Pi, f_{i}$ and $\theta$. The main difference is that those maps are smooth.

${ }^{18}$ By a slight abuse of notation, we denote by $\Pi$ and $\Pi^{1}$ the extensions of those maps to $\mathcal{X}$ whereas they were originally defined on $\mathcal{U} \subset \mathcal{X}$. Both are defined with the same formulas as $\Pi$ (respectively $\Pi^{1}$ ). Nevertheless, note that these extensions are not proper, that is why we only consider here the inverse images of compact subsets of $\Omega$. At the end, we obtain the existence of an equilibrium only for positive endowments.
} 
Since $\Omega$ is connected, the degree does not depend on the choice of the element where it is computed. Let $\overline{\mathbf{e}} \in \Omega$ defined, for $i \in M$, by $\bar{e}_{i}:=f_{i}^{1}\left(p, w_{i}\right)$ for some $\left(p,\left(w_{i}\right)_{i=1}^{m}\right) \in \mathbb{S} \times \mathbb{R}_{++}^{m}$. Let $\bar{B}:=B_{c}(\overline{\mathbf{e}}, r)$ be a closed ball of center $\overline{\mathbf{e}}$ contained in $\Omega^{19}$ and $B:=B_{o}(\overline{\mathbf{e}}, r)$ the open ball of same center and same radius. We know that the set $F^{-1}(\bar{B})$ is a compact set. The set $F^{-1}(B)$ contains $F^{-1}(\{\overline{\mathbf{e}}\})$ and is an open set, by the continuity of $F$, contained in $F^{-1}(\bar{B})$. Hence $F^{-1}(\{\overline{\mathbf{e}}\})$ is contained in the interior of $F^{-1}(\bar{B})$.

We now define the set $\vartheta:=\operatorname{proj}_{\mathbb{S} \times \mathbb{R}_{++}^{m} \times\left(\mathbf{1}^{\perp}\right)^{m-1}} F^{-1}(B)$. This set is an open $\operatorname{set}^{20}$. The mapping $F$ is obviously continuous on $\bar{\vartheta} \times[0,1]$. Let $\hat{F}$ be the restriction of $F$ to $\bar{\vartheta} \times[0,1]$. From Balasko[1], the degree modulo 2 at $\overline{\mathbf{e}}$ of $F_{0}$ is equal to $1^{21}$. Since $F_{0}^{-1}(\{\overline{\mathbf{e}}\})$ is contained in $\vartheta$, the degree of $\hat{F}_{0}$ is also equal to the degree of $F_{0}$ by Property (d2) of the degree (See Deimling [12].). The degree of $\hat{F}_{1}$ at $\overline{\mathbf{e}}$ is also equal to 1 since $\hat{F}$ is a continuous homotopy. Since $F_{1}^{-1}(\{\overline{\mathbf{e}}\})$ is contained in $\vartheta$, the degree of $\Pi$ at $\overline{\mathbf{e}}$ is equal to the degree of $\hat{F}_{1}$. In conclusion, the degree of $\Pi$ is equal to 1 .

Hence, for all $\mathbf{e} \in \Omega, \Pi^{-1}(\{\mathbf{e}\}) \neq \emptyset$, which means that there exists an equilibrium for every economy $\mathbf{e} \in \Omega$. The third point is a consequence of the fact that $\Pi$ is locally $C^{1}$ around all the pre-images of a regular economy using that the degree of $\Pi$ is equal to 1 .

\section{Concluding remarks}

This paper provides a contribution in the analysis of the individual behavior and in the global analysis of the equilibrium with multiprior preferences. The first main result is that, under an assumption of linear independence of the priors, the demand of a consumer with multiprior preferences is locally Lipschitz and continuously differentiable on an open set of full Lebesgue measure. Using Rader [25] and this result, we derive that almost every economy has a finite number of equilibrium prices.

The second main result concerns the genericity of regular economies. We have recovered the usual results of the global analysis of economic equilibrium from a differentiable viewpoint. Beyond most of the previous contributions in non-smooth cases, we have obtained not only that almost every economy has a finite number of equilibrium prices but that there exists an open set of full Lebesgue measure on which the result holds true. Moreover, we have proven that the equilibrium price selections are continuously differentiable. Furthermore, regular economies enjoy good properties like the local constancy of the

\footnotetext{
${ }^{19}$ The radius $r$ has to be sufficiently small.

${ }^{20}$ The image of an open set by a projection map is open.

${ }^{21}$ See [1] pp 103-106.
} 
number of equilibrium prices.

The only restriction is the requirement of linear independence of the gradients. First note that this requirement is easy to check. Then, remark that this requirement is always satisfied when the agents have at most two "extremal" priors. It remains open to study cases where not all but just some of the "extremal" priors are linearly independent.

\section{Appendix}

Proof of Proposition 2.1 The assumptions on the functions $b_{i s}$ imply straightforwardly that the functions $\left(u_{i}^{k}\right)_{1 \leq k \leq n_{i}}$ satisfy Assumption 4 .

Let us prove that Assumption 5 is satisfied. Let $\bar{x} \in \mathbb{R}_{++}^{\ell}$.

If $\sum_{k \in M(\bar{x})} \gamma_{k} \nabla u_{i}^{k}(\bar{x})=0$ for some $\left(\gamma_{k}\right)_{k \in M(\bar{x})}$, then, for all $s \in\{1, \ldots, S\}$,

$$
\left(\sum_{k \in M(\bar{x})} \gamma_{k} \pi_{i}^{k}(s)\right) \nabla b_{i s}\left(\bar{x}_{s}\right)=0
$$

and for $s=0$,

$$
\left(\sum_{k \in M(\bar{x})} \gamma_{k}\right) \nabla b_{i 0}\left(\bar{x}_{0}\right)=0 .
$$

Since $\nabla b_{i s}\left(\bar{x}_{s}\right) \gg 0$ for all $s=0, \ldots, S$ by Assumption 2, we get for $s \in$ $\{1, \ldots, S\}$ :

$$
\sum_{k \in M(\bar{x})} \gamma_{k} \pi_{i}^{k}(s)=0
$$

By the linear independence of the probability vectors $\left(\pi_{i}^{k}\right)_{k=1}^{n}$, we conclude that $\gamma_{k}=0$ for $k \in M(\bar{x})$. Hence, the gradient vectors $\left(\nabla u_{i}^{k}(\bar{x})\right)_{k \in M(\bar{x})}$ are linearly independent. So, the functions $\left(u_{i}^{k}\right)_{1 \leq k \leq n_{i}}$ satisfy Assumption 5 .

By simple computation, Assumption 3 implies that Assumption 6 is satisfied.

Details of the proof of Proposition 3.1 Let us summarize what follows. First we present the first order conditions characterizing the demand $f(p, w)$. Then, we consider a compact neighborhood $\Xi$ of an arbitrary element $\left.(\bar{p}, \bar{w}) \in \mathbb{R}_{++}^{\ell} \times\right] 0,+\infty[$. We define a $\varepsilon$-budget set and also a demand function $f^{\varepsilon}$ corresponding to this budget set. We show that this demand function is singled valued and continuous on $\Xi$. Thirdly, we show that for some $\bar{\varepsilon}>0, f^{\bar{\varepsilon}}(p, w)$ satisfies the first order conditions characterizing $f(p, w)$ for every $(p, w) \in \Xi$. We conclude that $f(p, w)$ is a singleton for every 
$\left.(p, w) \in \mathbb{R}_{++}^{\ell} \times\right] 0,+\infty\left[\right.$ and that $f$ is continuous on $\left.\mathbb{R}_{++}^{\ell} \times\right] 0,+\infty[$ in Lemma 6.1. Finally, we show that $f$ is locally Lipschitz on $\left.\mathbb{R}_{++}^{\ell} \times\right] 0,+\infty[$ in Lemma 6.3. In Lemma 6.4, we show that the function $f$ is continuously differentiable locally around $(\bar{p}, \bar{w}) \in \Omega^{0}$.

First order characterization of Problem 3.2.

$f(p, w)$ is the solution of the problem:

$$
\left\{\begin{array}{l}
\min -t \\
\text { subject to } \\
t-u^{k}(x) \leq 0 \quad k=1, \ldots, n \\
p \cdot x-w \leq 0 \\
x \gg 0
\end{array}\right.
$$

Let us introduce the functions $\left.\tau: \mathbb{R} \times \mathbb{R}_{++}^{\ell} \times \mathbb{R}_{++}^{\ell} \times\right] 0,+\infty[\longrightarrow \mathbb{R}$ and $\left.g: \mathbb{R} \times \mathbb{R}_{++}^{\ell} \times \mathbb{R}_{++}^{\ell} \times\right] 0,+\infty\left[\longrightarrow \mathbb{R}^{n+1}\right.$ defined by:

- $\tau(t, x, p, w):=-t$,

- $g_{k}(t, x, p, w):=t-u^{k}(x)$ for $k \in\{1, \ldots, n\}$,

- $g_{n+1}(t, x, p, w):=p \cdot x-w$.

$(t, x)$ are the variables and $(p, w)$ the parameters. The gradients and the Hessian matrix with respect to $(t, x)$ are given for all $(t, x, p, w) \in \mathbb{R} \times \mathbb{R}_{++}^{\ell} \times$ $\left.\mathbb{R}_{++}^{\ell} \times\right] 0,+\infty[$ by:

- $\nabla \tau(t, x, p, w)=\left(\begin{array}{c}-1 \\ 0\end{array}\right)$,

- $\nabla g_{k}(t, x, p, w)=\left(\begin{array}{c}1 \\ -\nabla u^{k}(x)\end{array}\right)$ for $k \in\{1, \ldots, n\}$,

- $\nabla g_{n+1}(t, x, p, w)=\left(\begin{array}{l}0 \\ p\end{array}\right)$,

- $D^{2} \tau(t, x, p, w) \equiv 0$,

- $D^{2} g_{k}(t, x, p, w)=\left[\begin{array}{cc}0 & 0 \\ 0 & -D^{2} u^{k}(x)\end{array}\right]$ for $k \in\{1, \ldots, n\}$,

- $D^{2} g_{n+1}(t, x, p, w) \equiv 0$. 
The function $\tau$ is linear, the constraint functions $\left(g_{k}\right)_{k=1}^{n+1}$ are twice differentiable and satisfy: $\nabla g_{k}(t, x, p, w) \neq 0$ for all $k=1, \ldots, n+1$ and $(t, x, p, w) \in$ $\left.\mathbb{R} \times \mathbb{R}_{++}^{\ell} \times \mathbb{R}_{++}^{\ell} \times\right] 0,+\infty[$. The first order conditions related to this problem are: if $(t, x)$ is a solution, there exists $\lambda=\left(\lambda_{k}\right)_{1 \leq k \leq n+1} \in \mathbb{R}_{+}^{n+1}$ such that :

$$
\left\{\begin{array}{l}
\nabla \tau(t, x, p, w)+\sum_{k=1}^{n+1} \lambda_{k} \nabla g_{k}(t, x, p, w)=0 \\
\lambda_{k} g_{k}(t, x, p, w)=0 \forall k \in\{1, \ldots, n+1\} \\
g_{k}(t, x, p, w) \leq 0 \forall k \in\{1, \ldots, n+1\} \\
\left.(t, x, p, w) \in \mathbb{R} \times \mathbb{R}_{++}^{\ell} \times \mathbb{R}_{++}^{\ell} \times\right] 0,+\infty[
\end{array}\right.
$$

Note that we have: $\left\{k \in\{1, \ldots, n\} \mid g_{k}(t, x, p, w)=0\right\}=M(x)$. Thus, the multipliers $\lambda_{k}$ are equal to zero for $k \notin M(x)$. From the above formula for the gradients, these conditions can be rewritten as follows:

$$
\left\{\begin{array}{l}
\sum_{k=1}^{n} \lambda_{k}=1 \\
\lambda_{k}\left(t-u^{k}(x)\right)=0, k=1, \ldots, n \\
\lambda_{n+1}(p \cdot x-w)=0 \\
t-u^{k}(x) \leq 0, k=1, \ldots, n \\
p \cdot x-w \leq 0 \\
\lambda_{n+1} p=\sum_{k \in M(x)} \lambda_{k} \nabla u^{k}(x)
\end{array}\right.
$$

First order conditions are necessary and sufficient. The first order conditions are necessary because Mangasarian-Fromovitz condition for the qualification of the constraints is satisfied everywhere. Indeed, for $\alpha>0$ large enough, $(-\alpha,-\mathbf{1}) \cdot \nabla g_{k}(t, x, p, w)<0$ for all $k=1, \ldots, n+1$.

We now check that these conditions are sufficient. Let $(t, x, \lambda)$ satisfying these conditions. By the first equation and the fact that the vectors $\left(\nabla u^{k}(x)\right)_{k=1}^{n}$ and $p$ are elements of $\mathbb{R}_{++}^{\ell}$, we deduce that $\lambda_{n+1}$ is necessarily positive, which implies that the budget constraint is binding. If $(t, x)$ is not a solution of 6.1 , there exists $\left(t^{\prime}, x^{\prime}\right)$ such that $t^{\prime}>t, t^{\prime} \leq u^{k}\left(x^{\prime}\right)$ for $k=1, \ldots, n$ and $p \cdot x^{\prime} \leq w$. Since $u^{k}$ is strictly quasi-concave with a nonvanishing gradient, for all $k \in M(x)$, one has $t=u^{k}(x)<t^{\prime} \leq u^{k}\left(x^{\prime}\right)$, so $\nabla u^{k}(x) \cdot\left(x^{\prime}-x\right)>0$. Hence,

$$
\lambda_{n+1} p \cdot\left(x^{\prime}-x\right)=\left(\sum_{k \in M(x)} \lambda_{k} \nabla u^{k}(x)\right) \cdot\left(x^{\prime}-x\right)>0
$$

which implies that $p \cdot x^{\prime}>p \cdot x=w$ in contradiction with $p \cdot x^{\prime} \leq w$. So, the first order conditions are sufficient.

In the next lemma, we prove that $f(p, w)$ is a singleton and $f$ is continuous. 
Lemma 6.1 Under Assumptions 4, 5 and $6, f(p, w)$ is a singleton for all $p \in \mathbb{R}_{++}^{\ell}$ and $w>0$. The function $f$ is continuous on $\left.\mathbb{R}_{++}^{\ell} \times\right] 0,+\infty[$.

Since the budget set is not closed, we need first to consider an intermediary problem.

$A \varepsilon$-problem For $\varepsilon>0, f^{\varepsilon}(p, w)$ is defined as the solution of the following optimization problem:

$$
\left\{\begin{array}{l}
\max u(x) \\
\text { subject to } p \cdot x \leq w \\
x_{h} \geq \varepsilon, h=1, \ldots, \ell
\end{array}\right.
$$

We define the $\varepsilon$-budget set by:

$$
B^{\varepsilon}(p, w):=\left\{x \in \mathbb{R}^{\ell} \mid x_{h} \geq \varepsilon, h=1, \ldots, \ell, p \cdot x \leq w\right\}
$$

Lemma 6.2 For every $\left.(\bar{p}, \bar{w}) \in \mathbb{R}_{++}^{\ell} \times\right] 0,+\infty[$, there exist a number $\hat{\varepsilon}>0$ and a neighborhood $\Xi$ of $(\bar{p}, \bar{w})$ such that, for all $\varepsilon \in] 0, \hat{\varepsilon}[$, for all $(p, w) \in \Xi$, the set $f^{\varepsilon}(p, w)$ is a singleton and the function $f^{\varepsilon}$ is continuous on $\Xi$.

Proof of Lemma 6.2 Let $\left.(\bar{p}, \bar{w}) \in \mathbb{R}_{++}^{\ell} \times\right] 0,+\infty[$ and $\varepsilon>0$ smaller than $\hat{\varepsilon}:=\frac{\bar{w}}{2 \sum_{h=1}^{\ell} \bar{p}_{h}}$. Such a choice implies that the interior of the set $B^{\varepsilon}(p, w)$ is nonempty on a compact neighborhood $\Xi$ of $(\bar{p}, \bar{w})$.

For all $(p, w) \in \Xi$, the set $B^{\varepsilon}(p, w)$ is a nonempty, compact and convex set. Thus, by Weierstrass Theorem, the set $f^{\varepsilon}(p, w)$ is nonempty. By the strict quasi-concavity of the function $u^{22}$, the set $f^{\varepsilon}(p, w)$ is a singleton.

On $\Xi$, the interior of $B^{\varepsilon}(p, w)$ is nonempty. We now prove that the function $(p, w) \longmapsto f^{\varepsilon}(p, w)$ is continuous on $\Xi$. This is a consequence of Berge's Theorem[3] since the correspondence $B^{\varepsilon}$ is both upper semi-continuous and lower semi-continuous on $\Xi$.

On $\Xi$, the set $B^{\varepsilon}(p, w)$ remains in a fixed compact set $K$. Hence the upper semi-continuity of $B^{\varepsilon}$ is equivalent to the closedness of its graph, which is a consequence of the continuity of the budget constraint.

We now show that the correspondence $B^{\varepsilon}$ is lower semi-continuous. Let us first introduce the correspondence $\hat{B}^{\varepsilon}$ defined on $\Xi$ by: $\hat{B}^{\varepsilon}(p, w):=\{x \in$ $\left.\mathbb{R}^{\ell} \mid p \cdot x<w, x_{h}>\varepsilon, h=1, \ldots, \ell\right\}$. The correspondence $\hat{B}^{\varepsilon}$ has an open graph by the continuity of the constraints. So $\hat{B}^{\varepsilon}$ is lower semi-continuous. Since $\hat{B}^{\varepsilon}(p, w)$ is nonempty for every $(p, w) \in \Xi$ and the closure of $\hat{B}^{\varepsilon}(p, w)$ is $B^{\varepsilon}(p, w)$, we deduce that the correspondence $B^{\varepsilon}$ is lower semi-continuous

\footnotetext{
${ }^{22}$ Indeed a differentiably strictly quasi-concave function is strictly quasi-concave (See Balasko[2]). And the minimum of strictly quasi-concave functions is strictly quasi-concave.
} 
since the closure of a lower semi-continuous correspondence is lower semicontinuous $^{23}$.

Proof of Lemma 6.1. Since the function $u$ is strictly quasi-concave and the budget set is convex, $f(p, w)$ contains at most one element.

First we show that for some $\bar{\varepsilon}>0, f^{\bar{\varepsilon}}(p, w)$ satisfies the first order conditions characterizing $f(p, w)$ for every $(p, w) \in \Xi$. We conclude that $f(p, w)$ is a singleton for every $\left.(p, w) \in \mathbb{R}_{++}^{\ell} \times\right] 0,+\infty[$ and that $f$ is continuous on $\left.\mathbb{R}_{++}^{\ell} \times\right] 0,+\infty[$. To use the first order conditions, we rewrite Problem 6.4 as we did since the function $u$ is not differentiable. For all $\left.(p, w) \in \mathbb{R}_{++}^{\ell} \times\right] 0,+\infty[$, $f^{\varepsilon}(p, w)$ is the solution of the following problem:

$$
\left\{\begin{array}{l}
\min -t \\
\text { subject to } \\
g_{k}(t, x, p, w) \leq 0 k=1, \ldots, n+1 \\
a_{h}(t, x, p, w):=\varepsilon-x_{h} \leq 0 h=1, \ldots, \ell
\end{array}\right.
$$

This problem is similar to Problem 6.1 except that there are $\ell$ additional constraints, $\varepsilon-x_{h} \leq 0, h=1, \ldots, \ell$.

As before, the necessary first order conditions can be written: if $(t, x)$ is a solution of 6.5 , there exists $\lambda^{\varepsilon}=\left(\lambda_{k}^{\varepsilon}\right)_{0 \leq k \leq n+1} \in \mathbb{R}_{+}^{n+1}$ and $\mu^{\varepsilon} \in \mathbb{R}_{+}^{\ell}$ such that :

$$
\left\{\begin{array}{l}
\sum_{k=1}^{n} \lambda_{k}^{\varepsilon}=1 \\
\lambda_{k}^{\varepsilon}\left(t-u^{k}(x)\right)=0, k=1, \ldots, n \\
\lambda_{n+1}^{\varepsilon}(p \cdot x-w)=0 \\
t-u^{k}(x) \leq 0, k=1, \ldots, n \\
p \cdot x-w \leq 0 \\
\mu_{h}^{\varepsilon}\left(\varepsilon-x_{h}\right)=0, h=1, \ldots, \ell \\
\varepsilon-x_{h} \leq 0, h=1, \ldots, \ell \\
\lambda_{n+1}^{\varepsilon} p=\sum_{k \in M(x)} \lambda_{k}^{\varepsilon} \nabla u^{k}(x)+\mu^{\varepsilon}
\end{array}\right.
$$

Note that $\left\{k \in\{1, \ldots, n\} \mid g_{k}(t, x, p, w)=0\right\}=M(x)$. Thus, the multipliers $\lambda_{k}^{\varepsilon}$ are equal to zero for $k \notin M(x)$. Since $\lambda_{n+1}^{\varepsilon} \neq 0, p \cdot x=w$ and the choice of $\varepsilon$ small enough implies that at least one constraint $a_{\bar{h}}$ is not binding. The first order conditions are necessary because Mangasarian-Fromovitz qualification condition is satisfied everywhere. Indeed, for $\alpha>0$ large enough, $\left(-\alpha,-\mathbf{1}_{\bar{h}}\right) \cdot \nabla g_{k}(t, x, p, w)<0$ for all $k=1, \ldots, n+1^{24}$. The satiated additional constraints are affine and we have: $\left(-\alpha,-\mathbf{1}_{\bar{h}}\right) \cdot \nabla a_{h}(t, x, p, w)=0$.

Our goal is to show that there exists $\bar{\varepsilon}>0$ such that the multipliers related to the constraints $\left(a_{h}\right)_{h=1}^{\ell}$ are equal to zero for all $(p, w)$ in $\Xi$. We reason by

\footnotetext{
${ }^{23}$ See the appendix of [16].

${ }^{24} \mathbf{1}_{h}$ denotes the vector that all the components are equal to zero except the $h$-th one that is equal to one.
} 
contradiction. Otherwise, there would exist a decreasing sequence ${ }^{25}\left(\varepsilon_{q}\right)_{q \geq 0}$ that converges to zero and a sequence of $\Xi$ denoted by $\left(p_{q}, w_{q}\right)_{q \geq 0}$ such that $\mu^{\varepsilon_{q}}\left(p_{q}, w_{q}\right) \neq 0$.

By compactness, we remark that the sequence $\left(x_{q}:=f^{\varepsilon_{q}}\left(p_{q}, w_{q}\right)\right)_{q \geq 0}$ converges, up to a subsequence, to an element $\hat{x}$ belonging to the boundary of $\mathbb{R}_{++}^{\ell}$ and that the sequence $\left(p_{q}, w_{q}\right)_{q \geq 0}$ converges, up to a subsequence, to some element $(\hat{p}, \hat{w}) \in \Xi$. In particular, remark that $\hat{w}$ is positive and that $\hat{p}$ is different from zero. With a slight abuse of notation, we denote the converging subsequences as the original sequences. We write, for $q \in \mathbb{N}$ and $k \in\{1, \ldots, n\}, \alpha_{k}^{\varepsilon_{q}}:=\frac{\lambda_{k}^{\varepsilon_{q}}}{\lambda_{n+1}^{\varepsilon_{q}}}$. We have, for all $q \geq 0$,

$$
\left\{\begin{array}{l}
p_{q} \cdot x_{q}-w_{q}=0 \\
p_{q}=\sum_{k=1}^{n} \alpha_{k}^{\varepsilon_{q}} \nabla u^{k}\left(x_{q}\right)+\mu^{\varepsilon_{q}} \\
\mu_{h}^{\varepsilon_{q}}\left(\varepsilon_{q}-x_{q h}\right)=0, h=1, \ldots, \ell
\end{array}\right.
$$

$0 \leq \mu^{\varepsilon_{q}} \leq p_{q}$ implies that $\left(\mu^{\varepsilon_{q}}\right)_{q \geq 0}$ is a bounded sequence. Since all the terms are non-negative in the second equation, for all $q \in \mathbb{N}$, we have:

$$
\left\|p_{q}\right\| \geq \alpha_{k}^{\varepsilon_{q}}\left\|\nabla u^{k}\left(x_{q}\right)\right\| \forall k \in M\left(x_{q}\right) .
$$

Hence:

$$
0 \leq \alpha_{k}^{\varepsilon_{q}} \frac{\nabla u^{k}\left(x_{q}\right) \cdot x_{q}}{\left\|p_{q}\right\|} \leq \frac{\nabla u^{k}\left(x_{q}\right) \cdot x_{q}}{\left\|\nabla u^{k}\left(x_{q}\right)\right\|} .
$$

for all $k \in\{1, \ldots, n\}$ and $q \in \mathbb{N}$. From Assumption 6 , we obtain, for all $k \in\{1, \ldots, n\}$,

$$
\lim _{q \longrightarrow+\infty} \frac{\nabla u^{k}\left(x_{q}\right) \cdot x_{q}}{\left\|\nabla u^{k}\left(x_{q}\right)\right\|}=0 .
$$

So , for all $k \in\{1, \ldots, n\}$,

$$
\lim _{q \longrightarrow+\infty} \alpha_{k}^{\varepsilon_{q}} \frac{\nabla u^{k}\left(x_{q}\right) \cdot x_{q}}{\left\|p_{q}\right\|}=0 .
$$

Finally, from $6.7, \mu^{\varepsilon_{q}} \cdot x_{q}=\varepsilon_{q} \mu^{\varepsilon_{q}} \cdot \mathbf{1}=\varepsilon_{q}\left\|\mu^{\varepsilon_{q}}\right\|$ converges to zero. Hence, summing the above limits, $\frac{p_{q} \cdot x_{q}}{\left\|p_{q}\right\|}$ converges to zero which contradicts $\frac{\hat{p} \cdot \hat{x}}{\|\hat{p}\|}=$ $\frac{\hat{w}}{\|\hat{p}\|}>0$

\footnotetext{
${ }^{25} \varepsilon_{0}$ is supposed to be smaller than $\hat{\varepsilon}$.
} 
Therefore, there exists $\bar{\varepsilon}>0$ such that $\mu^{\bar{\varepsilon}}=0$. Thus, $f^{\bar{\varepsilon}}$ satisfies the necessary and sufficient conditions related to 6.5 for all $(p, w)$ in $\Xi$. So $f=f^{\bar{\varepsilon}}$ on $\Xi$ and the continuity of $f$ follows.

The following lemma is a consequence of the result of Cornet and Vial[8].

Lemma 6.3 The function $f$ is locally Lipschitz on $\left.\mathbb{R}_{++}^{\ell} \times\right] 0 ;+\infty[$. Hence $f$ is almost everywhere differentiable on $\left.\mathbb{R}_{++}^{\ell} \times\right] 0 ;+\infty[$.

Proof To show that the function $f$ is locally Lipschitz, we check that Assumptions (A.0), (C.1) and (C.2) of Corollary 2.3. of [8] are satisfied.

Assumptions (A.0) are satisfied. We take $U=\mathbb{R} \times \mathbb{R}_{++}^{\ell}$ and $P=$ $\left.\mathbb{R}_{++}^{\ell} \times\right] 0,+\infty[$. The set $U$ is open and obviously a metric space. So Assumption (A.0) (i) is satisfied. Assumptions (A.0) (ii), (iii), (iv) and (v) are satisfied because the functions are $C^{2}$ on the set $U \times P$. Assumption (A.0) (vi) is satisfied with $Q=C=-\mathbb{R}_{+}^{n+1}$.

Assumption (C.1) is satisfied. We show that, at a solution $(t, x)$ of 6.1 , the vectors $\left(\nabla g_{k}(t, x, p, w)\right)_{k \in M(x)}$ and $\nabla g_{n+1}(t, x, p, w)$ are linearly independent. Let a vector $\left(\left(\gamma_{k}\right)_{k \in M(x)}, \gamma_{n+1}\right) \in \mathbb{R}^{\sharp M(x)+1}$ such that:

$$
\sum_{k \in M(x)} \gamma_{k} \nabla g_{k}(t, x, p, w)+\gamma_{n+1} \nabla g_{n+1}(t, x, p, w)=0
$$

We obtain:

$$
\left\{\begin{array}{l}
\sum_{k \in M(x)} \gamma_{k}=0 \\
\gamma_{n+1} p=\sum_{k \in M(x)} \gamma_{k} \nabla u^{k}(x)
\end{array}\right.
$$

If $\gamma_{n+1}=0$, then $\gamma_{k}=0$ for every $k \in M(x)$ because the vectors $\left(\nabla u^{k}(x)\right)_{k \in M(x)}$ are linearly independent by Assumption 5 .

If $\gamma_{n+1} \neq 0$, we get by the first order conditions:

$$
\sum_{k \in M(x)}\left(\frac{\lambda_{k}}{\lambda_{n+1}}-\frac{\gamma_{k}}{\gamma_{n+1}}\right) \nabla u^{k}(x)=0 .
$$

So we have: $\frac{\lambda_{k}}{\lambda_{n+1}}=\frac{\gamma_{k}}{\gamma_{n+1}}$ for every $k \in M(x)$ by Assumption 5. But we get a contradiction since:

$$
0=\sum_{k \in M(x)} \frac{\gamma_{k}}{\gamma_{n+1}}=\sum_{k \in M(x)} \frac{\lambda_{k}}{\lambda_{n+1}}=\frac{1}{\lambda_{n+1}}>0 .
$$

Assumption (C.2) is satisfied. Let $(t, x)$ be a solution of 6.1 with a corresponding multiplier $\lambda:=\left(\lambda_{k}\right)_{k=1}^{n+1}$. Let us introduce the set $K(x):=\{k \in$ 
$\left.M(x) \mid \lambda_{k}>0\right\}$. We have to check that, for all $h \in \mathbb{R}^{\ell+1}, h \neq 0$ such that: $\nabla \tau(t, x, p, w) \cdot h=0$ and $\nabla g_{k}(t, x, p, w) \cdot h=0$ for $k \in K(x) \cup\{n+1\}$, we have:

$$
\left[D^{2} \tau(t, x, p, w)+\sum_{k \in K(x) \cup\{n+1\}} \lambda_{k} D^{2} g_{k}(t, x, p, w)\right] h \cdot h>0 .
$$

Since we have : $D^{2} \tau \equiv 0$ and $D^{2} g_{n+1} \equiv 0$. It remains to show that:

$$
\sum_{k \in K(x)} \lambda_{k} D^{2} g_{k}(t, x, p, w) h \cdot h>0 .
$$

This reduces to:

$$
-\sum_{k \in K(x)} \lambda_{k} D^{2} u^{k}(x) \hat{h} \cdot \hat{h}>0
$$

where $\hat{h}$ is the vector of $\mathbb{R}^{\ell}$ deduces from $h$ by deleting the first component. Since $\nabla \tau(t, x, p, w) \cdot h=0$ and $\nabla g_{k}(t, x, p, w) \cdot h=0$ for every $k \in K(x) \cup$ $\{n+1\}$, one obtains $h_{0}=0$ with the first equality and $\nabla u^{k}(x) \cdot \hat{h}=0$ for $k \in K(x)$. So $h \neq 0$ and the inequality is then a consequence of Assumption 4 .

From [8], the function $f$ is locally Lipschitz on $\left.\mathbb{R}_{++}^{\ell} \times\right] 0,+\infty[$ and by Rademacher's Theorem, the function $f$ is almost everywhere differentiable.

The following lemma is a consequence of the Implicit Function Theorem and its proof is quite standard borrowing ideas from Fiacco McCormick [15].

Lemma 6.4 If $(\bar{p}, \bar{w}) \in \Omega^{0}$, then the function $f$ and the multipliers are continuously differentiable on an open neighborhood $\mathcal{N}$ of $(\bar{p}, \bar{w})$. Moreover: $M[f(p, w)]=M[f(\bar{p}, \bar{w})]$ for all $(p, w) \in \mathcal{N}$. Consequently, $\Omega^{0}$ is an open set.

Proof Recall that we have: $\forall k \in M(f(\bar{p}, \bar{w})), \lambda_{k}(\bar{p}, \bar{w})>0$. In this proof, we use the following notations: $\bar{x}:=f(\bar{p}, \bar{w})$ and $\bar{\lambda}_{k}:=\lambda_{k}(\bar{p}, \bar{w})$ for $k \in$ $\{1, \ldots, n\}$. To simplify the notation, without loss of generality, we suppose that we have: $M(f(\bar{p}, \bar{w}))=\{1, \ldots, r\}$. Then, locally around $(\bar{p}, \bar{w})$, by the continuity of the demand function and of the functions $\left(u^{k}\right)_{1 \leq k \leq n}$, $(u(f(p, w), f(p, w))$ is the solution of the following problem:

$$
\left\{\begin{array}{l}
\min -t \\
\text { subject to } \\
t-u^{k}(x) \leq 0, k \in\{1, \ldots, r\} \\
p \cdot x-w \leq 0 \\
x \gg 0
\end{array}\right.
$$


As already shown, since the first order optimality conditions are necessary and sufficient, the element $(u(f(p, w)), f(p, w))$ and the related multipliers $\left(\lambda_{1}(p, w), \ldots, \lambda_{r}(p, w), \lambda_{n+1}(p, w)\right)$ are solution of $G(t, x, \lambda, p, w)=0$ where $G$ is defined by:

$$
G(t, x, \lambda, p, w)=\left\{\begin{array}{l}
\left(\sum_{k=1}^{r} \lambda_{k}\right)-1 \\
\sum_{k=1}^{r} \lambda_{k} \nabla u^{k}(x)-\lambda_{n+1} p \\
t-u^{k}(x), k=1, \ldots, r \\
p \cdot x-w
\end{array}\right.
$$

To show that the function $f$ and the multipliers are continuously differentiable on a neighborhood of $(\bar{p}, \bar{w})$, from the Implicit Function Theorem, it suffices to show that $A$, the partial Jacobian matrix of $G$ with respect to $(t, x, \lambda)$, has full column rank.

$$
A:=\left(\begin{array}{cccccc}
\mathbf{t} & \mathbf{x} & \lambda_{\mathbf{1}} & \ldots & \lambda_{\mathbf{r}} & \lambda_{\mathbf{n}+\mathbf{1}} \\
0 & 0 & 1 & \ldots & 1 & 0 \\
0 & \sum_{k=1}^{r} \bar{\lambda}_{k} D^{2} u^{k}(\bar{x}) & \nabla u^{1}(\bar{x}) & \ldots & \nabla u^{r}(\bar{x}) & -\bar{p} \\
1 & -\nabla u^{1}(\bar{x})^{T} & 0 & \ldots & \ldots & 0 \\
\vdots & \vdots & \vdots & \vdots & \vdots & \vdots \\
1 & -\nabla u^{r}(\bar{x})^{T} & 0 & \ldots & \ldots & 0 \\
0 & \bar{p}^{T} & 0 & \ldots & \ldots & 0
\end{array}\right)
$$

It is sufficient to prove that $A\left(\begin{array}{c}\Delta t \\ \Delta x \\ \Delta \lambda\end{array}\right)=0$ implies: $(\Delta t, \Delta x \Delta \lambda)=(0,0,0)$. $\Delta t$ is a real number, $\Delta x$ is a column vector of dimension $\ell$ and $\Delta \lambda$ is a column vector of dimension $r+1$.

We obtain the system:

$$
\begin{array}{r}
\sum_{k=1}^{r} \Delta \lambda_{k}=0 \\
\sum_{k=1}^{r}\left[\bar{\lambda}_{k} D^{2} u^{k}(\bar{x}) \Delta x+\Delta \lambda_{k} \nabla u^{k}(\bar{x})\right]-\Delta \lambda_{n+1} \bar{p}=0 \\
\Delta t-\Delta x \cdot \nabla u^{k}(\bar{x})=0 \forall k \in\{1, \ldots, r\} \\
\Delta x \cdot \bar{p}=0
\end{array}
$$

From Equations 6.11, for all $k \in\{1, \ldots, r\}, \bar{\lambda}_{k} \Delta t-\bar{\lambda}_{k} \Delta x \cdot \nabla u^{k}(\bar{x})=0$. Summing over $k$, we obtain:

$$
\left(\sum_{k=1}^{r} \bar{\lambda}_{k}\right) \Delta t-\Delta x \cdot\left(\sum_{k=1}^{r} \bar{\lambda}_{k} \nabla u^{k}(\bar{x})\right)=0
$$


Since $\bar{\lambda}_{n+1} \bar{p}=\sum_{k=1}^{r} \bar{\lambda}_{k} \nabla u^{k}(\bar{x})$, we get:

$$
\left(\sum_{k=1}^{r} \bar{\lambda}_{k}\right) \Delta t-\Delta x \cdot \bar{\lambda}_{n+1} \bar{p}=0
$$

From 6.12 and the fact that $\sum_{=1}^{r} \bar{\lambda}_{k}=1$, we deduce: $\Delta t=0$. Thanks to 6.11 ,

$$
\Delta x \cdot \nabla u^{k}(\bar{x})=0 \forall k \in\{1, \ldots, r\}
$$

which implies $\bar{p} \cdot \Delta x=0$ since $\bar{\lambda}_{n+1} \bar{p}=\sum_{k=1}^{r} \bar{\lambda}_{k} \nabla u^{k}(\bar{x})$ and $\bar{\lambda}_{n+1}>0$.

So if $\Delta x \neq 0$, from Assumption 4,

$$
\Delta x \cdot D^{2} u^{k}(\bar{x}) \Delta x<0 \forall k \in\{1, \ldots, r\} .
$$

Doing an inner product of 6.10 by $\Delta x$, we get:

$$
\sum_{k=1}^{r} \bar{\lambda}_{k} \Delta x \cdot D^{2} u^{k}(\bar{x}) \Delta x+\sum_{k=1}^{r}\left(\Delta \lambda_{k} \nabla u^{k}(\bar{x}) \cdot \Delta x\right)-\Delta \lambda_{n+1} \bar{p} \cdot \Delta x=0
$$

which becomes:

$$
\sum_{k=1}^{r} \bar{\lambda}_{k} \Delta x \cdot D^{2} u^{k}(\bar{x}) \Delta x=0
$$

which is in contradiction with $\Delta x \cdot D^{2} u^{k}(\bar{x}) \Delta x<0$ for all $k \in\{1, \ldots, r\}$ recalling that the multipliers $\bar{\lambda}_{k}$ are all positive. Hence, we get $\Delta x=0$.

Since $\bar{\lambda}_{n+1} \bar{p}=\sum_{k=1}^{r} \bar{\lambda}_{k} \nabla u^{k}(\bar{x}), 6.10$ becomes:

$$
\sum_{k=1}^{r}\left(\Delta \lambda_{k}-\frac{\Delta \lambda_{n+1}}{\bar{\lambda}_{n+1}} \bar{\lambda}_{k}\right) \nabla u^{k}(\bar{x})=0 .
$$

By Assumption 5, for every $k \in\{1, \ldots, r\}, \Delta \lambda_{k}=\frac{\Delta \lambda_{n+1}}{\bar{\lambda}_{n+1}} \bar{\lambda}_{k}$. From 6.9, we have:

$$
0=\sum_{k=1}^{r} \Delta \lambda_{k}=\sum_{k=1}^{r} \frac{\Delta \lambda_{n+1}}{\bar{\lambda}_{n+1}} \bar{\lambda}_{k}=\frac{\Delta \lambda_{n+1}}{\bar{\lambda}_{n+1}} .
$$

So $\Delta \lambda_{n+1}=0$ and finally, $\Delta \lambda_{k}=0$ for $k=1, \ldots, r$. $\square$

Proof of Lemma 3.2 We first recall that $D_{p} \Delta(\bar{p}, \bar{v})$ is the Hessian matrix of the expenditure function $e_{K}(., \bar{v})$. Since the map $e_{K}(., \bar{v})$ is concave, $D_{p} \Delta(\bar{p}, \bar{v})$ defines a symmetric negative semi-definite bilinear form.

For $p \in \mathbb{R}_{++}^{\ell}$ sufficiently near from $\bar{p}, \Delta_{K}(p, \bar{v})$ is characterized by the first order conditions: 
- $u^{k}\left(\Delta_{K}(p, \bar{v})\right)=\bar{v}^{k}, \forall k \in K$,

- $p=\sum_{k \in K} \mu_{k}(p) \nabla u^{k}\left(\Delta_{K}(p, \bar{v})\right)$ with $\mu_{k}(p)>0 \forall k \in K$.

We differentiate the first condition with respect to $p$ and obtain at $\bar{p}$ for all $q \in \mathbb{R}^{\ell}$ :

$$
\nabla u^{k}\left(\Delta_{K}(\bar{p}, \bar{v})\right) \cdot D_{p} \Delta_{K}(\bar{p}, \bar{v})(q)=\nabla u^{k}(\bar{x}) \cdot D_{p} \Delta_{K}(\bar{p}, \bar{v})(q)=0 \forall k \in K .
$$

These equalities tell us that the image of $D_{p} \Delta_{K}(\bar{p}, \bar{v})$ is contained in the linear subspace $\cap_{k \in K} \nabla u^{k}(\bar{x})^{\perp}$ of dimension $\ell-\sharp K^{26}$. Furthermore, since $D_{p} \Delta_{K}(\bar{p}, \bar{v})$ is negative semi-definite, $\nabla u^{k}(\bar{x})$ belongs to its kernel for all $k \in K$. Thus, the dimension of the image of $D_{p} \Delta_{K}(\bar{p}, \bar{v})$ is at most $\ell-\sharp K$. We differentiate the second condition with respect to $p$. We have for $q \in \mathbb{R}^{\ell}$ :

$q=\sum_{k \in K} \mu_{k}(p) D^{2} u^{k}\left(\Delta_{K}(p, \bar{v})\right) D_{p} \Delta_{K}(p, \bar{v})(q)+\sum_{k \in K}\left(\nabla \mu_{k}(p) \cdot q\right) \nabla u^{k}\left(\Delta_{K}(p, \bar{v})\right)$.

For all $q \in \cap_{k \in K} \nabla u^{k}(\bar{x})^{\perp}$, we have:

$$
q=\left[\sum_{k \in K} \mu_{k}(\bar{p}) D^{2} u^{k}(\bar{x})\right] D_{p} \Delta_{K}(\bar{p}, \bar{v})(q) .
$$

So we have for $q \in \cap_{k \in K} \nabla u^{k}(\bar{x})^{\perp}$ :

$$
D_{p} \Delta_{K}(\bar{p}, \bar{v})(q)=0 \Longrightarrow q=0 .
$$

So the kernel of the restriction on $\cap_{k \in K} \nabla u^{k}(\bar{x})^{\perp}$ of $D_{p} \Delta_{K}(\bar{p}, \bar{v})$ is reduced to zero. So the rank of $D_{p} \Delta_{K}(\bar{p}, \bar{v})$ is at least $\ell-\sharp K$. Hence, the rank of $D_{p} \Delta_{K}(\bar{p}, \bar{v})$ is equal to $\ell-\sharp K$ and the kernel of $D_{p} \Delta_{K}(\bar{p}, \bar{v})$ is equal to $\mathcal{L}\left(\nabla u^{k}(\bar{x}), k \in K\right)$

Proof of Lemma 3.3 By Walras law, $p \cdot \bar{f}_{K}(p)=p \cdot \bar{x}$.

Differentiating with respect to $p$, we obtain for all $q \in \mathbb{R}^{\ell}$ :

$$
q \cdot \bar{f}_{K}(p)+p \cdot D \bar{f}_{K}(p)(q)=q \cdot \bar{x} .
$$

Since $\bar{x}=\bar{f}_{K}(\bar{p})$, this implies: $\bar{p} \cdot D \bar{f}_{K}(\bar{p})(q)=0$. Let $k \in K$. By the chain rule: $\nabla \hat{v}_{K}^{k}(p) \cdot q=\nabla u^{k}\left(\bar{f}_{K}(p)\right) \cdot D \bar{f}_{K}(p)(q)$.

\footnotetext{
${ }^{26}$ This is a consequence of Assumption 5. Indeed, $K \subset M(\bar{x})$ implies that the family $\left(\nabla u^{k}(\bar{x})\right)_{k \in K}$ is linearly independent.
} 
From the first order conditions of the utility maximization problem, we have:

$$
\sum_{k \in K} \bar{\lambda}_{k} \nabla u^{k}(\bar{x})=\bar{\lambda}_{n+1} \bar{p}
$$

Recalling that $\bar{f}(\bar{p})=\bar{x}$, we have:

$$
\begin{aligned}
\sum_{k \in K} \bar{\lambda}_{k} \nabla \hat{v}_{K}^{k}(\bar{p}) \cdot q & =\left(\sum_{k \in K} \bar{\lambda}_{k} \nabla u^{k}(\bar{x})\right) \cdot D \bar{f}_{K}(\bar{p})(q) \\
& =\bar{\lambda}_{n+1} \bar{p} \cdot D \bar{f}_{K}(\bar{p})(q)=0
\end{aligned}
$$

Since the equality is true for all $q \in \mathbb{R}^{\ell}$, we conclude that $\sum_{k \in K} \bar{\lambda}_{k} \nabla \hat{v}_{K}^{k}(\bar{p})=$ 0 . Recalling that the multipliers $\left(\bar{\lambda}_{k}\right)_{k \in K}$ are all positive and sum to 1 , since the functions $\left(\hat{v}_{K}^{k}\right)_{k \in K}$ coincide on a neighborhood of $\bar{p}$, one concludes $\nabla \hat{v}_{K}^{k}(\bar{p})=0$ for all $k \in K$.

Now we prove the second result. Since $\bar{f}_{K}(p)=\Delta_{K}\left(p, \hat{v}_{K}(p)\right)$ in an open neighborhood of $\bar{p}$, it suffices to use the chain rule for differential mappings and the above claim to conclude. Indeed, let $q \in \mathbb{R}^{\ell}$,

$$
D \bar{f}_{K}(\bar{p})(q)=D_{p} \Delta_{K}(\bar{p}, \bar{v})(q)+\left(\sum_{k \in K} \nabla \hat{v}_{K}^{k}(\bar{p}) \cdot q\right) D_{v} \Delta_{K}(\bar{p}, \bar{v}) .
$$

Proposition 6.1 Let $E$ be a vector space and $\Phi$ be a symmetric positive definite bilinear form on E. Let $\left(\xi_{i}\right)_{i=1}^{k}$ be a linearly independent family of vectors of $E$. There exists $\alpha \in R_{++}^{k}$ such that for all $i=1, \ldots k$,

$$
\Phi\left(\xi_{i}, \sum_{j=1}^{k} \alpha_{j} \xi_{j}\right)>0
$$

Let $S$ be the simplex of $R^{k}$ and $C$ the subset of $R^{k}$ defined by:

$$
C=\left\{\left(\Phi\left(\xi_{i}, \sum_{j=1}^{k} \alpha_{j} \xi_{j}\right)\right)_{i=1}^{k} \mid \alpha \in S\right\}
$$

Since $\Phi$ is bilinear, its restriction to the space generated by the family $\left(\xi_{i}\right)_{i=1}^{k}$ is continuous, so $C$ is a nonempty compact polyhedral subset of $R^{k}$.

Let us first show that the conclusion of the proposition holds true if $C \cap R_{++}^{k}$ is nonempty. Indeed, if there exists $c \in C \cap R_{++}^{k}$, then there exists $\alpha \in S$ such that for all $i, c_{i}=\Phi\left(\xi_{i}, \sum_{j=1}^{k} \alpha_{j} \xi_{j}\right)>0$. Since $\Phi$ is continuous on the space generated by the family $\left(\xi_{i}\right)_{i=1}^{k}$, there exists $t>0$ small enough 
so that for all $i, \Phi\left(\xi_{i}, \sum_{j=1}^{k}\left(\alpha_{j}+t\right) \xi_{j}\right)>0$, hence $\left(\alpha_{j}+t\right)_{j=1}^{k} \in R_{++}^{k}$ and the conclusion of the proposition holds true.

We now prove by contraposition that $C \cap R_{++}^{k}$ is nonempty. If it is not true, we apply a separation theorem between $C$ and $R_{++}^{k}$, so there exists an element $\lambda \in R^{k} \backslash\{0\}$ such that for $(c, d) \in C \times R_{++}^{k}, \lambda \cdot c \leq \lambda \cdot d$. Using usual arguments, one deduces that $\lambda \in R_{+}^{k}$ and $\lambda \cdot c \leq 0$ for all $c \in C$. Let $\bar{\alpha}=\left(1 / \sum_{j=1}^{k} \lambda_{j}\right) \lambda$. Then $\bar{\alpha} \in S$. Let $\bar{c}=\left(\Phi\left(\xi_{i}, \sum_{j=1}^{k} \bar{\alpha}_{j} \xi_{j}\right)\right)_{i=1}^{k}$, then $\bar{c} \in C$. We remark that:

$$
\begin{aligned}
\lambda \cdot \bar{c} & =\sum_{i=1}^{k} \lambda_{i} \Phi\left(\xi_{i}, \sum_{j=1}^{k} \bar{\alpha}_{j} \xi_{j}\right)=\Phi\left(\sum_{i=1}^{k} \lambda_{i} \xi_{i}, \sum_{j=1}^{k} \bar{\alpha}_{j} \xi_{j}\right) \\
& =\left(1 / \sum_{j=1}^{k} \lambda_{j}\right) \Phi\left(\sum_{i=1}^{k} \lambda_{i} \xi_{i}, \sum_{j=1}^{k} \lambda_{j} \xi_{j}\right) .
\end{aligned}
$$

So $\Phi\left(\sum_{i=1}^{k} \lambda_{i} \xi_{i}, \sum_{j=1}^{k} \lambda_{j} \xi_{j}\right) \leq 0$ and since $\Phi$ is positive definite, this implies that $\sum_{j=1}^{k} \lambda_{j} \xi_{j}=0$. Hence, since the family $\left(\xi_{i}\right)_{i=1}^{k}$ is linearly independent, one concludes that $\lambda_{j}=0$ for $j=1, \ldots, k$, which contradicts $\lambda \neq 0$.

\section{References}

[1] Balasko, Y.: Foundations of general equilibrium theory. Academic Press, New York (1988)

[2] Balasko, Y.: General equilibrium: Theory of value. Princeton University Press, Princeton (2011)

[3] Berge, C.: Espaces topologiques, fonctions multivoques. Dunod, Paris (1966)

[4] Biheng, N.: A generalized expenditure function. Documents de travail du Centre d'Economie de la Sorbonne 2014.83, Université Paris 1 (2014)

[5] Bonnisseau, J.M., Rivera-Cayupi, J.: Constrained consumptions, Lipschitzian demands and regular economies. Journal of Optimization Theory and Applications 131, 179-193 (2006)

[6] Carlier, G., Dana, R., Shahdi. N.: Efficient insurance contracts under epsilon contaminated utilities. The Geneva Papers on risk and Insurance Theory 28, 59-71 (2003)

[7] Chen, Z., Epstein, L.: Ambiguity, risk and asset returns in continuous time. Econometrica 70, 1403-1443 (2002)

[8] Cornet, B., Vial, J.P.: Lipschitzian solutions of perturbed non linear problems. SIAM Journal of control and optimization 24, 1123-1137 (1986) 
[9] Dana, R.A.: Ambiguity, uncertainty aversion and equilibrium welfare. Economic Theory 23, 569-587 (2004)

[10] Debreu, G.: Theory of Value. Yale University Press (1959)

[11] Debreu, G.: Economies with a finite set of equilibria. Econometrica 38, 387-392 (1970)

[12] Deimling, K.: Nonlinear functional analysis. Springer Verlag, Berlin Heidelberg (1985)

[13] Epstein, L., Wang, T.: Intertemporal asset pricing and Knightian uncertainty. Econometrica 62, 283- 322 (1994)

[14] Federer, H.: Geometric measure theory. Grundlehren der mathematischen Wissenschaften, Vol. 153. Springer Verlag, Berlin Heidelberg New York (1969)

[15] Fiacco, A., McCormick, G.: Nonlinear programming: Sequential unconstrained minimization techniques. Classics in Applied Mathematics, Vol. 4. SIAM, Philadelphia (1990)

[16] Florenzano, M.: General equilibrium analysis: Existence and optimality properties of equilibria. Kluwer Academic Publishers (2003)

[17] Gilboa, I., Schmeidler, D.: Maxmin expected utility with non-unique prior. Journal of Mathematical Economics 18, 141-153 (1989)

[18] Karni, E.: Decision-making under Uncertainty: The Case of StateDependent Preferences. Harvard University Press, Cambridge (1985)

[19] Karni, E., Schmeidler, D., Vind K.: On state dependent preferences and subjective probabilities. Econometrica 51, 1021-1031 (1983)

[20] Kopylov. I.: Subjective probability and confidence. Mimeo, University of California at Irvine, (2008)

[21] Magill, M., Quinzii, M.: Theory of Incomplete Markets. MIT Press, Cambridge (1996)

[22] Mas-Colell, A.: The theory of general economic equilibrium: A differentiable approach. Cambridge University Press, Cambridge (1985)

[23] Nishimura, K.G.,Ozaki, H.: Search and Knightian Uncertainty. Journal of Economic Theory 119, 299-333 (2004) 
[24] Nishimura, K.G.,Ozaki, H.: An axiomatic approach to $\varepsilon$-contamination. Economic Theory 27, 303-340 (2006)

[25] Rader, R.: Nice demand functions. Econometrica 41, 913-935 (1973)

[26] Rigotti, L., Shannon, C.: Sharing risk and ambiguity. Journal of Economic Theory 147, 2028-2039 (2012)

[27] Shannon, C.: Regular nonsmooth equations. Journal of Mathematical Economics 23, 147-166 (1994)

[28] Schmeidler, D.: Subjective probability and expected utility without additivity. Econometrica 57, 571-587 (1989)

[29] Shapley, L.S.: Core of a convex game. International Journal of Game Theory 1, 12-26 (1989)

[30] Wakker, P.: Subjective probabilities for state-dependent continuous utility. Mathematical Social Sciences 14, 289-298 (1987) 